Chapman University

Chapman University Digital Commons

\title{
Affect Variability and Predictability: Using Recurrence Quantification Analysis to Better Understand How the Dynamics of Affect Relate to Health
}

Brooke N. Jenkins

John F. Hunter

Michael J. Richardson

Tamlin S. Conner

Sarah D. Pressman

Follow this and additional works at: https://digitalcommons.chapman.edu/psychology_articles

Part of the Health Psychology Commons, Other Psychology Commons, and the Personality and Social Contexts Commons 


\section{Affect Variability and Predictability: Using Recurrence Quantification Analysis to Better Understand How the Dynamics of Affect Relate to Health}

\section{Comments}

This is a pre-copy-editing, author-produced PDF of an article accepted for publication in Emotion, volume 20 , issue 3 , in 2020 following peer review. The definitive publisher-authenticated version is available online at https://doi.org/10.1037/emo0000556.

\section{Copyright}

American Psychological Association 
Affect Variability and Predictability: Using Recurrence Quantification Analysis to Better Understand How the Dynamics of Affect Relate to Health 
Abstract

Changes in affect over time have been associated with health outcomes. However, previously utilized measurement methods focus on variability of affect (e.g., standard deviation, root mean squared successive difference) and ignore the more complex temporal patterns of affect over time. These patterns may be an important feature in understanding how the dynamics of affect relate to health. Recurrence quantification analysis (RQA) may help alleviate this problem by assessing temporal characteristics unassessed by past methods. RQA metrics, such as determinism and recurrence, can provide a measure of the predictability of affect over time, indexing how often patterns within affective experiences repeat. In Study 1, we first contrasted RQA metrics with commonly used measures of variability to demonstrate that RQA can further differentiate among patterns of affect. In Study 2, we analyzed the associations between these new metrics and health, namely, depressive and somatic symptoms. We found that RQA metrics predicted health above and beyond mean levels and variability of affect over time. The most desirable health outcomes were observed in people who had high mean positive affect, low mean negative affect, low affect variability, and high affect predictability. These studies are the first to demonstrate the utility of RQA for determining how temporal patterns in affective experiences are important for health outcomes.

Key words: recurrence quantification analysis, affect variability, affect predictability, depressive symptoms, somatic symptoms 
Affect Variability and Predictability: Using Recurrence Quantification Analysis to Better Understand How the Dynamics of Affect Relate to Health

Affective experiences are dynamic in nature. Feelings fluctuate from moment to moment and are intricately interconnected in a complex temporal system (Ebner-Priemer, Eid, Kleindienst, Stabenow, \& Trull, 2009; Pressman, Jenkins, Kraft-Feil, Rasmussen, \& Scheier, 2017). Many studies have demonstrated that average affect is linked to a variety of psychological and physical health and health-relevant outcomes (e.g., Chida \& Steptoe, 2008; Jenkins, Granger, et al., 2018; Pressman \& Cohen, 2005; Pressman, Jenkins, \& Moskowitz, 2019), but, increasingly, studies have revealed that the variation in affect over time may further predict health and health-relevant factors. For example, greater levels of affect variability, assessed with the standard deviation, have been associated with less favorable daily cortisol trajectories (Human et al., 2015) and worse immunocompetence (Jenkins, Hunter, Cross, Acevedo, \& Pressman, 2018). These findings mirror patterns in mental health outcomes, which typically find greater affect variability in people with higher levels of depressive symptoms, greater anxiety, and/or poorer psychological well-being (Gruber, Kogan, Quoidbach, \& Mauss, 2013; Peeters, Berkhof, Delespaul, Rottenberg, \& Nicolson, 2006). Therefore, it is critical that researchers be equipped with the proper tools to accurately assess the dynamics of affect. However, current measurements of affect dynamics fail to capture important information about changes over time because of their limited ability to assess the temporal structure within complex patterns of affective change.

The most common metric used to assess affect variability is the standard deviation (Röcke, Li, \& Smith, 2009). This approach captures affect variability as a single value by calculating the standard deviation of an individual's scores on an affect measure over multiple 
time points (Eid \& Diener, 1999; Gruber, Kogan, Quoidbach, \& Mauss, 2013; Hardy \& Segerstrom, 2016; Ram \& Gerstorf, 2009; Röcke \& Brose, 2013). An advantage of this approach is that standard deviation is easily understood and the value can be used as a predictor or outcome variable in subsequent models (Röcke et al., 2009). Nevertheless, this single value assumes an independence of assessment such that an assessment at time, $t$, is not necessarily related to time, $t+1$ (Ram \& Gerstorf, 2009). Assessing only the standard deviation offers information about the magnitude of the change in affect but provides no information about the sequential dependence or deterministic predictability of affective experiences.

More recently, researchers have begun using the root mean squared successive difference (RMSSD; Ebner-Priemer et al., 2009). This metric captures the degree of change in affect from one time point to the next by squaring all successive differences, averaging these squared differences, and then taking the square root of that average. RMSSD takes into account temporal ordering by reflecting the relative instability of affective change from one time point to the next. Nevertheless, RMSSD does not capture patterning of change over time beyond average change among two time points. A similar limitation occurs when measuring emotional inertia, which is often operationalized as the degree of autocorrelation between consecutive time points (Kuppens, Allen, \& Sheeber, 2010).

Information about the dynamics of affect over large time spans can provide more detailed insights over and above current affect variability measures. For example, an individual who has more structured or "predictable" negative affect (NA) values that denote a more recurrent pattern of affective experiences over time (i.e., exhibits a less stochastic and more stationary or periodic structure of affective change over time) may have much different outcomes compared to an individual who has "less predictable" values of NA that create a more inconsistent (i.e., 
stochastic) or less recurrent pattern of affect (see Figure 1). The predictability of fluctuations in affective experiences over time (i.e., regularity and consistency) may matter more than (or in addition to) simply assessing the magnitude of those fluctuations. Broadening the quantification of change to include metrics of predictability in addition to variability may allow researchers to better understand the full dynamics of affective experience over time.

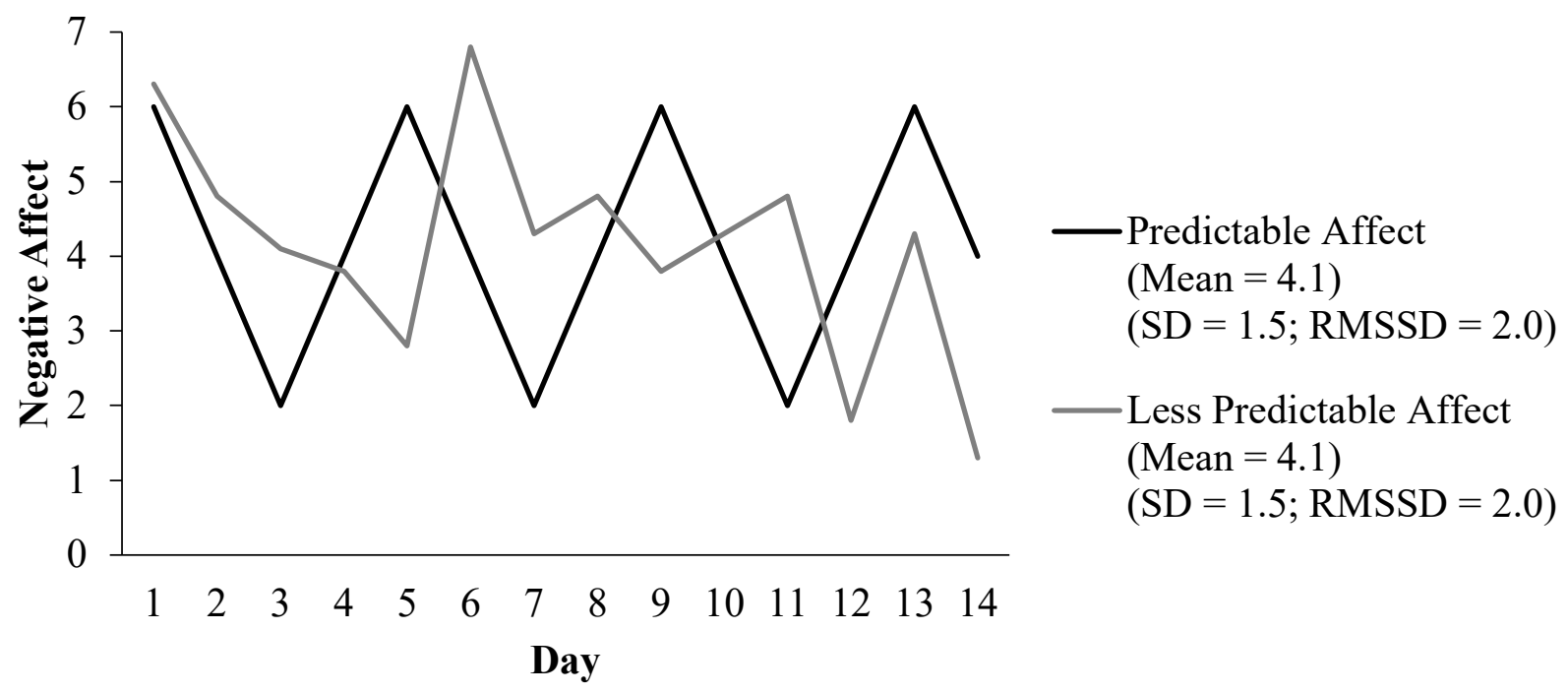

Figure 1. Two individuals with different predictability of affect but with identical means and variability. $\mathrm{SD}=$ standard deviation. $\mathrm{RMSSD}=$ root mean squared successive difference.

Recurrence quantification analysis (RQA) may be one such method for assessing patterns of affect predictability. RQA is a non-linear event- or time-series analysis method that assesses the dynamics of temporal sequences of change over time without researchers having to make any a priori assumptions about the nature of the dynamics that define a given behavioral event- or time-series recording. Although RQA has not been previously used to examine intraindividual changes in affect over time, it has been successfully employed to investigate a wide range of other dynamic human behaviors (e.g., eye gaze [Anderson et al., 2013], posture changes [Riley 
\& Clark, 2003], and affect synchrony between mother-child dyads [Main, Paxton, \& Dale, 2016]). Because of the temporal nature of affect, RQA lends itself nicely to studying dynamics of affect and, in particular, the degree of affect predictability (Richardson, Dale, \& Marsh, 2014). RQA provides multiple metrics of predictability, two of the most common being the percentage of deterministic structure $(\% \mathrm{DET})$ within a measured series and recurrence rate (i.e., percent recurrence, $\%$ REC). \%DET measures the degree to which recurrent states exhibit regular or structured patterns of change over time (i.e., repeated sequences of recurrent states). With regard to how affect changes over time, \%DET captures the degree to which the same (or similar) sequences of affective change occur over time, such that more structured or "predictable" patterns of affective change over time should result in high levels of \%DET. \%REC is a measure of state regularity which, in terms of affect, reflects how often a person experiences the same (or similar) level of affect over time (i.e., the degree to which the same state of affect reoccurs over time). Although \%DET and \%REC may provide different information for longer time-series data (e.g., over 50 time points), they are often correlated for shorter time-series (e.g., 15 time points).

Of particular relevance to the current study is the fact that the RQA metrics of \%DET and $\%$ REC should provide more information about the structural dynamics of affective change than standard (linear) variability statistics. In other words, the benefit of these RQA metrics with regard to understanding the complex dynamics of affect is that they can quantify the predictability of affect over time. Although previous research has demonstrated that greater affect variability (e.g., higher standard deviation) has negative implications for health (e.g., Gruber et al., 2013), no previous research has examined whether higher affect predictability may be beneficial for health. Specifically, the regularity or predictability of how affect changes over time may allow individuals to better prepare and then cope with affective experiences. For 
example, an individual who knows that NA is always high Monday mornings may be better prepared to cope with such NA. Thus, it is important to test how mean levels of affect, affect variability, and affect predictability interact, as these factors may operate together with health implications.

The purpose of the current research was to demonstrate the validity of the RQA method for quantifying the structure or predictability of affective time-series using simulated data (Study 1) and then apply RQA to a large, real data set (Study 2). In Study 1, the RQA metrics of \%DET and \%REC were compared to common variability metrics (standard deviation, RMSSD) using simulated data. This simulation compared predictability and variability metrics and determined whether each could add independent information about the patterns of affect across time (i.e., days). Specifically, we hypothesized that the same values of standard deviation or RMSSD would be associated with, but not differentiate between, patterns that are more or less predictable (i.e., stochastic and/or periodic), but that patterns could be quantifiably differentiated using the RQA metrics of \%DET and \%REC. In Study 2, the RQA metrics of \%DET and \%REC were then used, along with mean levels and variability, to predict health outcomes. We hypothesized that, as in previous literature, more variability would be associated with worse psychological and physical health outcomes (i.e., more depressive and somatic symptoms). In contrast, we also hypothesized that predictability would be associated with better psychological and physical health outcomes (i.e., fewer depressive and somatic symptoms).

\section{Study 1}

\section{Method}

Data simulation. Affect data were simulated by creating 14 instances for 900 cases, thus emulating typical daily dairy data collected once a day for two weeks with 900 participants. 
Within these 900 cases, nine distinct groups of 100 cases each were created to alter variability and predictability (see Table 1). Data were first simulated using the Stata 15 (StataCorp, 2017) generate function to randomly draw values from normal distributions. This altered the variability. Groups 1 through 3 had integer values generated from a normal distribution with a mean of 3.00 and a standard deviation of 0.25 (see Table 1 column 3). Groups 4 through 6 had integer values generated from a normal distribution with a mean of 3.00 and a standard deviation of 0.50 . Groups 7 through 9 had integer values generated from a normal distribution with a mean of 3.00 and a standard deviation of 1.00. Generating the integer valued time-series in this way ensured that Groups 1 through 3 would have small standard deviations (i.e., low variability), Groups 4 through 6 would have medium standard deviations (i.e., medium variability), and Groups 7 through 9 would have large standard deviations (i.e., high variability).

In addition to altering the variability of integer values, some cases had value sequences repeated (see Table 1 column 4) to alter predictability of the data, such that the data series contained levels of periodic structure. Repeating the values ensured that there would be greater predictability within these groups of data series, with greater levels of repeated (periodic) structure corresponding to higher predictability. One third of the groups had no values repeated (i.e., low predictability). One third of the groups had instances 1 through 7 repeated once (i.e., medium predictability). One third of the groups had instances 1 and 2 repeated 7 times (i.e., high predictability). It is important to note that this method of generating the data was not specific to a particular affect measure per se, but was employed to simply represent different amounts of variability and predictability that might be associated with changes in affect over time when rated on continuous scales (e.g., changes in positive affect (PA) or NA across a 14 day daily diary period). 
Table 1

Data Simulation Parameters and Predicted Results.

\begin{tabular}{|c|c|c|c|c|c|}
\hline \multirow[t]{2}{*}{ Group } & \multirow[t]{2}{*}{ Group Name } & \multirow{2}{*}{$\begin{array}{c}\text { Normal } \\
\text { Distribution } \\
\text { (mean, SD) }\end{array}$} & \multirow[t]{2}{*}{ \# of Days Repeated } & \multicolumn{2}{|c|}{ Predictions } \\
\hline & & & & Variability & Predictability \\
\hline 1 & $\begin{array}{l}\text { Low Variability - } \\
\text { Low Predictability }\end{array}$ & $(3,0.25)$ & none & Low & Low \\
\hline 2 & $\begin{array}{l}\text { Low Variability - } \\
\text { Medium Predictability }\end{array}$ & $(3,0.25)$ & $\begin{array}{c}7 \text { (first } 7 \text { are repeated } \\
\text { a second time) }\end{array}$ & Low & Medium \\
\hline 3 & $\begin{array}{l}\text { Low Variability - } \\
\text { High Predictability }\end{array}$ & $(3,0.25)$ & $\begin{array}{l}12 \text { (first } 2 \text { are repeated } \\
7 \text { more times) }\end{array}$ & Low & High \\
\hline 4 & $\begin{array}{l}\text { Medium Variability - } \\
\text { Low Predictability }\end{array}$ & $(3,0.5)$ & none & Medium & Low \\
\hline 5 & $\begin{array}{l}\text { Medium Variability - } \\
\text { Medium Predictability }\end{array}$ & $(3,0.5)$ & $\begin{array}{c}7 \text { (first } 7 \text { are repeated } \\
\text { a second time) }\end{array}$ & Medium & Medium \\
\hline 6 & $\begin{array}{l}\text { Medium Variability - } \\
\text { High Predictability }\end{array}$ & $(3,0.5)$ & $\begin{array}{c}12 \text { (first } 2 \text { are repeated } \\
7 \text { more times) }\end{array}$ & Medium & High \\
\hline 7 & $\begin{array}{l}\text { High Variability - } \\
\text { Low Predictability }\end{array}$ & $(3,1)$ & none & High & Low \\
\hline 8 & $\begin{array}{l}\text { High Variability - } \\
\text { Medium Predictability }\end{array}$ & $(3,1)$ & $\begin{array}{c}7 \text { (first } 7 \text { are repeated } \\
\text { a second time) }\end{array}$ & High & Medium \\
\hline 9 & $\begin{array}{l}\text { High Variability - } \\
\text { High Predictability }\end{array}$ & $(3,1)$ & $\begin{array}{c}12 \text { (first } 2 \text { are repeated } \\
7 \text { more times) }\end{array}$ & High & High \\
\hline
\end{tabular}




\section{Measures.}

Mean. Means were calculated within individuals by summing the values for each of the 14 days and then dividing by 14. Each participant's mean therefore represents their average score over the entire daily diary period.

Variability. Variability was assessed by standard deviation and RMSSD. Standard deviations were calculated within individuals by summing the squared distances for each day from the overall mean and then averaging those squared distances. RMSSD was calculated by squaring all successive differences, averaging them together, and then taking the square root of that average. The following formulas were used:

$$
\begin{gathered}
S D=\sqrt{\frac{\sum_{i=1}^{n}\left(x_{i}-\bar{x}\right)^{2}}{n}} \\
R M S S D=\sqrt{\frac{\sum_{i=2}^{n}\left(x_{i}-x_{i-1}\right)^{2}}{n-1}}
\end{gathered}
$$

Predictability. Predictability was measured using recurrence quantification analysis (RQA). The RQA measures of percent determinism (\%DET) and percent recurrence (\%REC) were calculated using the RQA software developed by Richardson, Riley, Shockley, and Dale (APA ATI, 2015; see Supplemental Material A for a detailed description of how to compute $\%$ DET and \%REC). Given that integer value time-series data were investigated here and in Study 2, a form of RQA known as Categorical-RQA was employed. As illustrated in Figure 2, this method of RQA first involves identifying reoccurring (recurrent) values within a discrete time-series by plotting them on a 2-dimensional recurrence plot (Figure 2). Essentially, a data time-series is represented on both the $x$ and $y$ axis of a 2-dimensional grid, with recurrent points indicating when the same value within the data series reoccurs. For example, a row of simulated 
data is presented in Figure 2. Recurrent states (i.e., "points") within the recurrence plot correspond to when the same value reoccurs indicating that the same level of affect that was previously experienced has reoccurred. Note that given that each value within the data series is recurrent with itself, the main diagonal of an (auto-) recurrence plot (i.e., single time-series recurrence plot), also known as the line of identity, is ignored. That is, \%DET and \%REC quantifications exclude recurrent states along the main diagonal. 


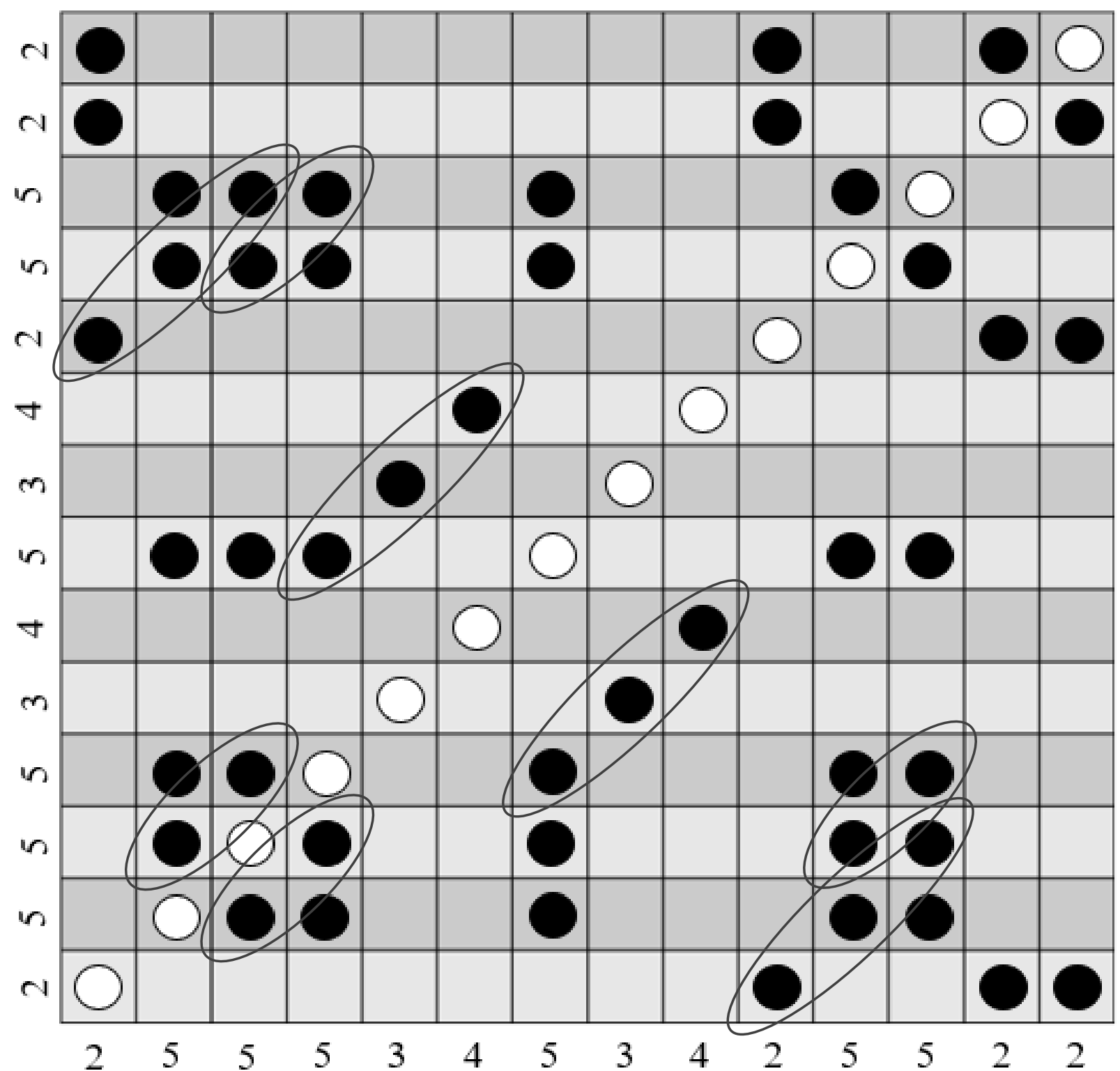

Figure 2. Data analysis for RQA. The same data time-series is placed on the $\mathrm{x}$ - and $\mathrm{y}$-axis.

Recurrent states or "points" are placed where same values intersect. For example, in the lower left corner a "point" is placed because there is a 2 on the $\mathrm{x}$-axis and a 2 on the $y$-axis. Points that are circled are those that lie on a diagonal line. Only points that form diagonal lines (excluding the line of identity; the white points) that contain two or more recurrent points are used in the determinism calculation. 
In short, \%DET equals the percentage of recurrent points that form diagonal lines (in this case 20; excluding the line of identity) of the total number of points (in this case 46) within a recurrence plot, where a diagonal line corresponds to two or more consecutive recurrent points. For instance, in Figure 2, \%DET $=20 / 46=.43 \rightarrow 43 \%$. With regard to an affect data series, $\% \mathrm{DET}$ represented the percentage of time an individual experiences the same pattern of change in affect over time and, therefore, the degree of predictability or deterministic structure within an affect data series. \%REC is calculated by dividing the number recurrent points that do not fall along the main diagonal (in this case 46) by the number of spaces (in this case 182). In Figure 2, $\%$ REC $=46 / 182=.25 \rightarrow 25 \% . \%$ REC indexes the proportional degree to which an individual experiences the same measured states of affect over time. Note that although $\%$ REC and $\%$ DET often co-vary, $\%$ REC does not provide a measure of predictability on its own, but only in relation to \%DET. For instance, randomly shuffling the example time-series in Figure 2 would not alter $\%$ REC, but would significantly affect $\%$ DET. That is, $\%$ DET has the potential to provide the best picture of the degree to which the states of a system or data series repeated the same sequential dependent sequences over time (i.e., are governed by a deterministic or predictable dynamic process).

Statistical approach. Analysis of variance (ANOVA) and post hoc pairwise comparisons with a Bonferroni correction for familywise error were used to assess differences in the metrics (i.e., mean, standard deviation, RMSSD, \%DET, and \%REC) among the 9 groups. $95 \%$ confidence intervals are presented for mean values. Effect sizes of $\eta^{2}$ and their corresponding $90 \%$ confidence intervals ${ }^{1}$ are presented (Steiger, 2004). A power analysis conducted using G*Power (Faul, Erdfelder, Buchner, \& Lang, 2009) revealed that a sample size

\footnotetext{
${ }^{1}$ Due to the one-tailed probability test of ANOVA, $90 \%$ confidence intervals should be used to ensure that confidence intervals do not include 0 when p-values are less than .05 (Steiger, 2004).
} 
of 100 per group would be sufficient to detect a medium effect size with statistical power to evaluate this hypothesis at the 0.95 level.

\section{Results}

Figure 3 presents a visual depiction of the mean, variability, and predictability measures for each of the 9 groups. Analysis of variance results indicated that there were no overall mean differences among the 9 groups $F(8,891)=0.45, p=.891, \eta^{2}<0.01,90 \%$ CI $[0.00,0.00]$ (see Table 2). As can be seen in Figure 3a, the mean was relatively stable over the 9 groups. 

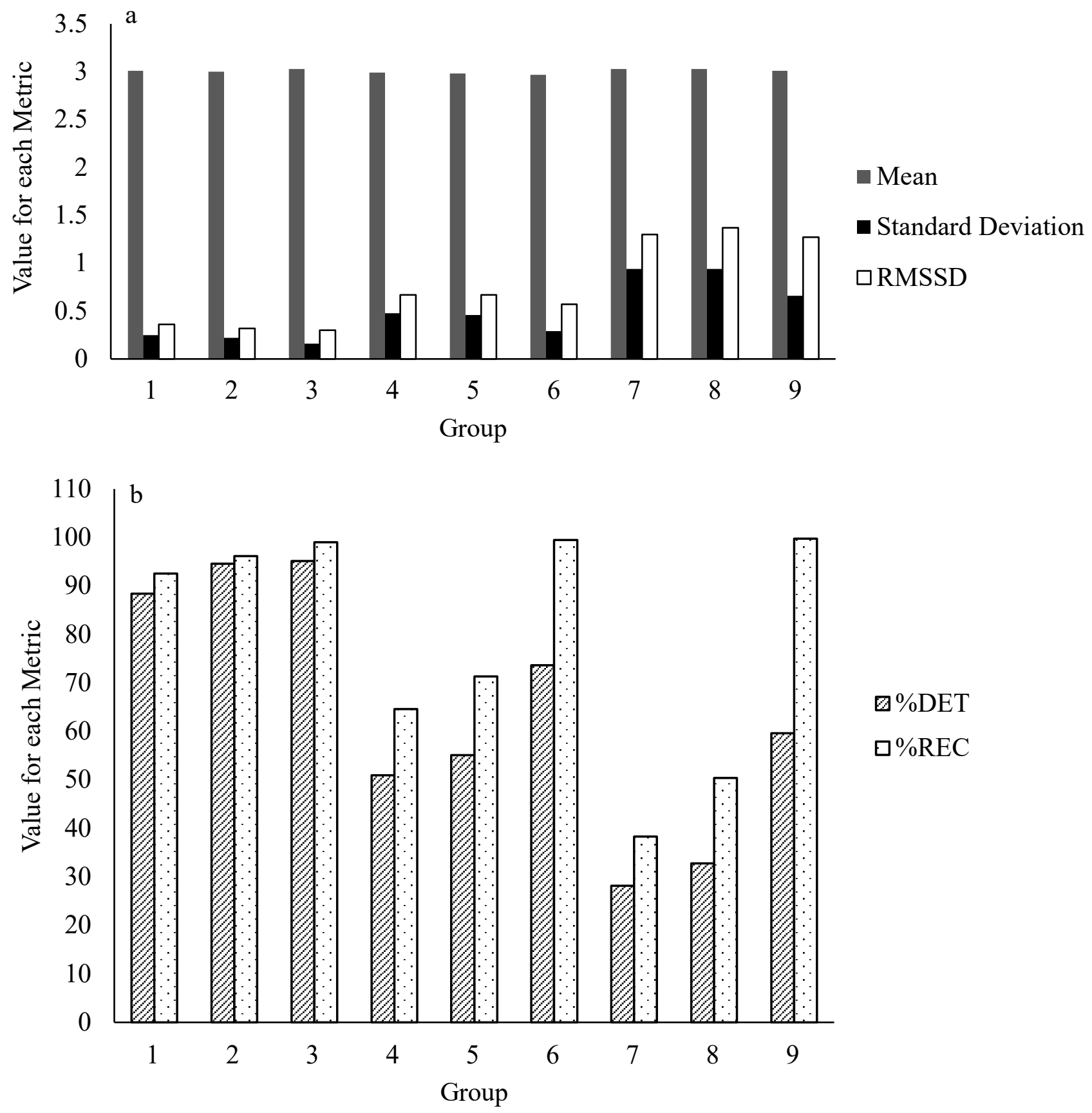

Figure 3. Mean, standard deviation, root mean squared successive difference (RMSSD), percent determinism $(\% \mathrm{DET})$, and percent recurrence $(\% \mathrm{REC})$ values by group. Note that the mean is about the same across all groups while the standard deviation and RMSSD is similar for groups 1 through 3, then 4 through 6 , and then 7 through 9. \%DET and \%REC help distinguish within these sets of groups. 
Table 2

Mean, Standard Deviation, RMSSD, and Recurrence Quantification Measures by Group.

\begin{tabular}{|c|c|c|c|c|c|c|}
\hline \multirow[b]{2}{*}{ Group } & \multirow[b]{2}{*}{ Group Name } & \multirow[t]{2}{*}{ Mean } & \multicolumn{2}{|c|}{ Variability } & \multicolumn{2}{|c|}{ Predictability } \\
\hline & & & Standard & RMSSD & $\% \mathrm{DET}$ & $\% \mathrm{REC}$ \\
\hline & & & Deviation & & & \\
\hline \multirow[t]{2}{*}{1} & Low Variability - & $3.01^{\mathrm{a}}$ & $0.25^{\mathrm{ab}}$ & $0.36^{\mathrm{a}}$ & $92.60^{\mathrm{a}}$ & $88.41^{\mathrm{a}}$ \\
\hline & Low Predictability & $(3.00,3.02)$ & $(0.24,0.26)$ & $(0.34,0.38)$ & $(90.95,94.26)$ & $(86.14,90.67)$ \\
\hline \multirow[t]{2}{*}{2} & Low Variability - & $3.00^{\mathrm{a}}$ & $0.22^{\mathrm{ab}}$ & $0.32^{\mathrm{a}}$ & $96.13^{\mathrm{ab}}$ & $94.55^{\mathrm{a}}$ \\
\hline & Medium Predictability & $(2.98,3.02)$ & $(0.21,0.23)$ & $(0.30,0.34)$ & $(94.75,97.52)$ & $(92.23,96.87)$ \\
\hline \multirow[t]{2}{*}{3} & Low Variability - & $3.03^{\mathrm{a}}$ & $0.16^{b}$ & $0.30^{\mathrm{a}}$ & $99.00^{\mathrm{b}}$ & $95.15^{\mathrm{a}}$ \\
\hline & High Predictability & $(2.99,3.06)$ & $(0.13,0.18)$ & $(0.26,0.35)$ & $(98.94,99.06)$ & $(92.08,98.23)$ \\
\hline \multirow[t]{2}{*}{4} & Medium Variability - & $2.99^{a}$ & $0.48^{\mathrm{c}}$ & $0.67^{b}$ & $64.57^{\mathrm{c}}$ & $50.99^{\mathrm{bc}}$ \\
\hline & Low Predictability & $(2.96,3.01)$ & $(0.46,0.50)$ & $(0.65,0.70)$ & $(61.25,67.90)$ & $(48.30,53.68)$ \\
\hline \multirow[t]{2}{*}{5} & Medium Variability - & $2.98^{\mathrm{a}}$ & $0.46^{\mathrm{c}}$ & $0.67^{b}$ & $71.30^{\mathrm{d}}$ & $55.08^{b}$ \\
\hline & Medium Predictability & $(2.95,3.02)$ & $(0.43,0.49)$ & $(0.62,0.72)$ & $(67.51,75.08)$ & $(51.26,58.89)$ \\
\hline \multirow[t]{2}{*}{6} & Medium Variability - & $2.97^{\mathrm{a}}$ & $0.29^{\mathrm{a}}$ & $0.57^{\mathrm{b}}$ & $99.44^{\mathrm{b}}$ & $73.62^{d}$ \\
\hline & High Predictability & $(2.90,3.04)$ & $(0.25,0.34)$ & $(0.48,0.65)$ & $(99.33,99.55)$ & $(68.25,78.98)$ \\
\hline \multirow[t]{2}{*}{7} & High Variability - & $3.03^{\mathrm{a}}$ & $0.94^{\mathrm{d}}$ & $1.30^{\mathrm{c}}$ & $38.30^{\mathrm{e}}$ & $28.13^{\mathrm{e}}$ \\
\hline & Low Predictability & $(2.98,3.08)$ & $(0.90,0.98)$ & $(1.23,1.37)$ & $(35.30,41.31)$ & $(26.66,20.60)$ \\
\hline \multirow[t]{2}{*}{8} & High Variability - & $3.03^{\mathrm{a}}$ & $0.94^{\mathrm{d}}$ & $1.37^{\mathrm{c}}$ & $50.41^{\mathrm{f}}$ & $32.75^{\mathrm{e}}$ \\
\hline & Medium Predictability & $(2.95,3.10)$ & $(0.88,0.99)$ & $(1.29,1.46)$ & $(47.05,53.77)$ & $(30.74,34.75)$ \\
\hline \multirow[t]{2}{*}{9} & High Variability - & $3.01^{\mathrm{a}}$ & $0.66^{\mathrm{e}}$ & $1.27^{\mathrm{c}}$ & $99.73^{b}$ & $59.62^{c}$ \\
\hline & High Predictability & $(2.87,3.15)$ & $(0.56,0.76)$ & $(1.08,1.47)$ & $(99.63,99.82)$ & $(54.97,64.27)$ \\
\hline
\end{tabular}

Note. Column values with similar letters indicate no significant difference $(p>.05) .95 \%$ confidence intervals are presented in parentheses. RMSSD = root mean squared successive difference. $\% \mathrm{DET}=$ percent determinism. $\% \mathrm{REC}=$ percent recurrence. 
Analysis of variance results indicated that there were overall differences among the 9 groups for the variability measures (standard deviation: $F(8,891)=163.77, p<.001, \eta^{2}=0.60$, 90\% CI [0.56, 0.62]; RMSSD: $F(8,891)=106.21, p<.001, \eta^{2}=0.49,90 \%$ CI $\left.[0.45,0.52]\right)$. Variability metrics tended to be in line with predictions such that groups 1 through 3 had the lowest values while groups 7 through 9 had the highest values (see Figure 3a and Table 2).

Analysis of variance results also indicated that there were overall differences among the 9 groups for the predictability measures $\left(\% \mathrm{DET}: F(8,891)=392.96, p<.001, \eta^{2}=0.78,90 \% \mathrm{CI}\right.$ $[0.76,0.79] ; \%$ REC: $F(8,891)=230.63, p<.001, \eta^{2}=0.67,90 \%$ CI $\left.[0.65,0.69]\right)$. The $\%$ DET and \%REC measures followed similar patterns as the hypothesized results (see Figure $3 b$ ). Looking within low (groups 1 through 3), medium (groups 4 through 6), and high (groups 7 through 9) variability groups, \%DET and \%REC tended to become larger as the data were repeated more often (i.e., more predictable). For example, \%DET grew from 64.57 to 71.30 to 99.44 across groups 4 through 6 respectively (see Figure $3 \mathrm{~b}$ and Table 2). Although the standard deviation was held constant for these groups, more instances of repeated data (i.e., predictability) occurred for group 6 compared to group 5 and more repeated data occurred for group 5 compared to group 4. However, when the standard deviation was smaller (i.e., groups 1 through 3), there was less discrepancy between $\%$ REC and \%DET values among the groups. For example, the pairwise comparisons between groups 1 and 2 and groups 2 and 3 were no longer significantly different, $p \mathrm{~s}>.05$ (see Table 2). Nevertheless, the same pattern of results occurred throughout the data whereby as predictability increased, so did \%DET and \%REC values.

\section{Discussion}

The results demonstrate that the RQA metrics of \%DET and \%REC can be employed to index the dynamics of variables like affect and provide different and new pieces of information 
about how affect changes over time compared to traditional measures of variability. RQA further differentiates simulated cases based on metrics of predictability that consider the role of time in assessing patterns of affective experiences. Over the 9 groups, mean levels were stable and did not distinguish between the groups. And, as noted by the other measures (standard deviation, RMSSD, \%DET, \%REC), the same mean level of affect can be associated with different levels of variability and predictability. This demonstrates that studies relying only on mean level of affect across time may be overlooking important information.

In addition, these results demonstrate that relying solely on the standard deviation or RMSSD approach for assessing affective change is insufficient for capturing the finer details about the patterns of variation across time. For groups 4 through 6 (see Figure 3a), RMSSD (along with the means) was kept constant. Based on these values, previous researchers may have assumed that each of these groups were equal. However, once the RQA measures are taken into consideration, a substantial difference in affective patterning emerges and it becomes apparent that these groups are in fact not equal. When the same patterns of affect are repeated more often, there is an increase in \%DET and \%REC (see Figure 3b). For example, group 4 and group 6 have nearly identical means and RMSSD as one another. However, group 4 is different from group 6 because the same pattern of affect was repeated 7 times (i.e., more predictability), as noted by $\% \mathrm{DET}$ and \%REC. Repeated patterns, and the predictability that follows, vary from person to person and are indicative of individual differences in affective experiences. This is critical because those differences in patterns of variation may have distinct implications for various outcomes. The addition of RQA metrics adds more information about the dynamic nature of affect and demonstrates how the relation between affective experiences over time may influence how affective profiles are categorized and understood. 
There are several limitations to Study 1. First, although the normal distributions were intended to mirror similar means and standard deviations appearing in the affect literature (e.g., Jenkins et al., 2018), the smaller and larger standard deviations may be less likely. Similarly, the repeated nature of the data may or may not be ecologically valid. It is likely that repeated affect could occur from week to week with, for example, affect on Mondays looking similar to affect on other Mondays and affect on Fridays looking similar to affect on other Fridays. Therefore, the medium repeated data (in which data from 7 days are repeated across the next 7 days) may closely reflect this week by week repeated structure. Additionally, a no repeated condition (low repeat) was included which would mirror the natural environment if affect did not follow a specific repeated structure. It is important to note that even when data were not purposefully repeated, random repeated days could have occurred.

Regardless of these limitations, this study demonstrates that RQA measures may add more detailed information above and beyond measures of variability. Thus, the next step is to explore if this new methodology offers additional explanatory power in terms of its association with real world outcomes. If RQA helps determine the nonlinear dynamics that underlie affective change, researchers can potentially (a) build dynamical models of affective change and make corresponding predictions from that mode and (b) develop a machine-learning model or nonlinear function approximator (i.e., neural network) model to predict affective change. However, the key for either modelling approach is to first identify the degree to which there is a deterministic process underlying affective change (even if this process is chaotic or aperiodic). This latter goal is the aim of Study 2. 


\section{Study 2}

Building on the findings of Study 1, in Study 2 we link affect mean, variability, and predictability to depressive and somatic symptom reports as most previous studies assessing affect variability have concentrated on similar outcomes (Gruber et al., 2013; Houben, Van Den Noortgate, \& Kuppens, 2015; Human et al., 2015; Peeters et al., 2006). Capturing averages, variability, and predictability of affective experiences may provide researchers with a better understanding of how the intricacies of the affective experience influence mental and physical health. Furthermore, since these factors are not perfectly correlated, it may be advantageous to understand how they interact to predict certain health outcomes. The initial simulation study (Study 1) provided a foundational rationale for why these factors are important, and the following study (Study 2) applied the RQA methodology to real health outcomes.

\section{Method}

Participants. Study 2 used data from the "Daily Life Study" conducted from 2011 to 2014 at the University of Otago, New Zealand. Participants included 1,482 college students $\left(M_{\text {age }}=19.76, S D_{\text {age }}=2.43\right)$. Sixty-seven percent of the participants were female. Participants were 78\% Caucasian, 10\% Asian, 5\% Māori/Pacific Islander, 3\% Indian, and 4\% were another ethnicity or mixed ethnicity.

Procedure. Participants completed an initial survey asking about demographics and depressive symptoms. Participants then completed daily diaries for 13 consecutive days. The diaries consisted of several questionnaires including affect and stress measures. After the 13 consecutive days, participants then completed a follow up survey asking about physical health symptoms. All study procedures were approved by the University of Otago, New Zealand Ethics Committee. 
Affect mean, variability, and predictability. State emotion adjectives were assessed each day for 13 days on a scale from 1 (Not at all) to 5 (Extremely) describing how much each of the words reflected how the participant felt that day. Nine NA words (nervous, dejected, irritable, hostile, sad, angry, unhappy, anxious, and tense) were averaged to create a daily NA value (Cronbach's alpha range for each of the 13 days $=.87$ to .91 ) and nine PA words (happy, excited, cheerful, pleasant, calm, energetic, enthusiastic, content, and relaxed) were averaged to create a daily PA value (Cronbach's alpha range for each of the 13 days $=.88$ to .92 ). Affect words were selected to capture a range of high to low intensities on the affective circumplex (Barrett \& Russell, 1999). Day averages were then averaged over the 13 days to create an overall PA and NA mean value. Then, standard deviations and RMSSD values over the 13 time points were calculated for PA and NA with the formulas used in Study 1 to create measures of variability ${ }^{2}$. Finally, the RQA metrics, \%DET and \%REC, were calculated using the methods and software described in Study 1 to create measures of predictability. All PA and NA daily mean values were rounded to the nearest integer value to allow for Categorical-RQA. These calculations resulted in mean, variability, and predictability values: NA mean (NAMEAN), PA mean (PAMEAN), NA standard deviation (NASD), PA standard deviation (PASD), NA RMSSD (NARMSSD), PA RMSSD (PA RMSSD), NA \%DET (NA\%DET), PA \%DET (PA\%DET), NA \%REC (NA\%REC), and PA \%REC (PA\%REC). \%REC and \%DET were divided by 100 to be similar in range to the NA and PA means, standard deviations, and RMSSD values.

Distress. Distress was assessed each day with the question "Overall, how much stress (e.g., because of hassles, demands, or other stressors) have you been under today?" rated on a

\footnotetext{
${ }^{2}$ We reanalyzed the data using autocorrelation as a measure of emotional inertia (see Supplemental Material B). The pattern of results remained similar with the exception of a few deviations. We describe these in Supplemental Material B.
} 
scale of 0 (no stress) to 4 (a great deal of stress). The response for each day was averaged over the 13 days and used as a control variable in all analyses as has been done in previous research on affect variability (e.g., Gruber et al, 2013).

Depressive symptoms. The Center for Epidemiologic Studies Depression Scale (CES-D; Radloff, 1977), a 20 item measure, was used to assess depressive symptoms in the initial survey. Items included statements such as "I did not feel like eating; my appetite was poor," "I thought my life had been a failure," and "My sleep was restless." Items were scored using the following scale to assess how often the items reflected how they felt during the past week: rarely or none of the time (0), some or a little of the time (1), occasionally or a moderate amount of the time (2), or most or all of the time (3) (positive items reverse scored). Scores were summed with higher values indicating higher levels of depressive symptoms (Cronbach's alpha $=.89$ ).

Self-reported somatic symptoms. During the follow up survey, participants were asked whether they felt like they had a cold or flu in the past two weeks (rated on a scale from $0=$ not at all to 4 = very). Additionally, they were asked whether they "felt physically 'run down'," "felt tired," and "felt refreshed when [they] woke up in the mornings" (each rated on a scale from $0=$ not at all to 4 = very; the refreshed item was reverse coded). These four items were summed, with higher values reflecting a greater number of self-reported somatic symptoms.

Statistical approach. Depressive and somatic symptoms were used as the dependent variables in all analyses. Due to the dependent variables being count variables and skewed, poisson regression in Stata 15 (StataCorp, 2017) was used. Mean, variability, and predictability and the interactions among them were used as independent variables in a series of 10 models. Models 1 through 5 reflect the NA results while models 6 through 10 reflect the PA results. Model 1 used mean and variability as the independent variables of interest. Model 2 added the 
mean*variability interaction term to Model 1. Model 3 added the predictability metric to Model 2. Model 4 added the mean*predictability interaction term to Model 4. Model 5 added the variability*predictability and the mean*variability*predictability interaction terms to Model 4. Models 6 through 10 mirrored Models 1 through 5 but used the PA variables. All predictor variables were mean centered to allow for ease of interpretation in interaction terms. Distress was adjusted for in all models. Effect sizes as well as 95\% confidence intervals are presented throughout.

\section{Results}

Descriptive statistics. Table 3 presents the means and standard deviations of the affect metrics as well as their associations. PA mean was higher than NA mean $(t(1,298)=59.90, p<$ $.001,95 \%$ CI of the difference $[1.29,1.37]$, Cohen's $d=2.74,95 \%$ CI of Cohen's $d[2.63$, 2.85]). PA was more variable compared to NA as evidenced by the variability measures (PASD vs. NA $A_{\mathrm{SD}}, t(1,298)=15.44, p<.001,95 \% \mathrm{CI}$ of the difference $[0.08,0.10]$, Cohen's $d=0.45$, 95\% CI of Cohen's $d[0.38,0.53]$; PARMSSD Vs. NARMSSD, $t(1,298)=13.87, p<.001,95 \%$ CI of the difference $[0.09,0.12]$, Cohen's $d=0.41,95 \%$ CI of Cohen's $d[0.33,0.49])$. NA was more predictable compared to PA as evidenced by the RQA measures (NA\%DET Vs. PA\%DET, $t(1,298)=$ $11.52, p<.001,95 \%$ CI of the difference [6.18, 8.72], Cohen's $d=0.40,95 \%$ CI of Cohen's $d$ $[0.32,0.48]$; NA\%REC Vs. PA\%REC, $t(1,298)=12.41, p<.001,95 \%$ CI of the difference $[6.73$, 9.25], Cohen's $d=0.42,95 \%$ CI of Cohen's $d[0.34,0.49])$. Interestingly, NA mean and affect variability measures were all positively associated while NA mean was negatively associated with affect predictability measures. In other words, individuals higher in NA were more likely to have variable affect but less likely to have predictable affect. On the other hand, PA mean was positively associated with affect predictability measures and negatively associated with affect 
variability measures. These PA findings indicate that individuals higher in PA have more predictable, but less variable affect.

The variability metrics of standard deviation and RMSSD were very highly correlated with each other (NA: $r=0.90, p<.001,95 \%$ CI $[0.89,0.91]$; PA: $r=0.82, p<.001,95 \% \mathrm{CI}$ $[0.80,0.84]$; see Table 3$)$. This was also true for the predictability metrics (NA: $r=0.84, p<$ $.001,95 \%$ CI [0.82, 0.86]; PA: $r=0.80, p<.001,95 \%$ CI $[0.78,0.82]$; see Table 3$).$ No other correlations between any of the variables were higher than these associations. Therefore, for ease of presenting the results, the following analyses use standard deviation as the metric for variability and \%DET as the metric for predictability. Standard deviation and $\% \mathrm{DET}$ were selected over RMSSD because they resulted in greater differentiation among the 9 groups in Study 1 (see Table 2). 
Table 3

Mean, Standard Deviation, and Pearson's Correlation of Affect Metrics.

\begin{tabular}{|c|c|c|c|c|c|c|c|c|c|c|c|}
\hline & Mean & SD & $\mathrm{NA}_{\mathrm{SD}}$ & $\mathrm{NA}_{\mathrm{RMSSD}}$ & NA\%DET & $\mathrm{NA}_{\% \mathrm{REC}}$ & PAMEAN & $\mathrm{PA}_{\mathrm{SD}}$ & $\mathrm{PA}_{\mathrm{RMSSD}}$ & $\mathrm{PA}_{\% \mathrm{DET}}$ & $\mathrm{PA}_{\% \mathrm{REC}}$ \\
\hline NAMEAN & 1.67 & 0.47 & $0.64 * * *$ & $0.57 * * *$ & $-0.52 * * *$ & $-0.59 * * *$ & $-0.36 * * *$ & $0.17 * * *$ & $0.16 * * *$ & $-0.12 * * *$ & $-0.12 * * *$ \\
\hline $\mathrm{NA}_{\mathrm{SD}}$ & 0.41 & 0.22 & & $0.90 * * *$ & $-0.66 * * *$ & $-0.76 * * *$ & $-0.24 * * *$ & $0.44 * * *$ & $0.39 * * *$ & $-0.30 * * *$ & $-0.32 * * *$ \\
\hline $\mathrm{NA}_{\mathrm{RMSSD}}$ & 0.52 & 0.28 & & & $-0.65 * * *$ & $-0.69 * * *$ & $-0.21 * * *$ & $0.40 * * *$ & $0.44 * * *$ & $-0.27 * * *$ & $-0.29 * * *$ \\
\hline NA\%DET & 71.67 & 19.03 & & & & $0.84 * * *$ & $0.26 * * *$ & $-0.29 * * *$ & $-0.29 * * *$ & $-0.22 * * *$ & $-0.23 * * *$ \\
\hline NA\%REC & 58.36 & 21.11 & & & & & $0.29 * * *$ & $-0.34 * * *$ & $-0.31 * * *$ & $0.25 * * *$ & $0.28 * * *$ \\
\hline PAMEAN & 3.00 & 0.50 & & & & & & $-0.12 * * *$ & $-0.10 * * *$ & $0.06 * * *$ & $0.09 * * *$ \\
\hline $\mathrm{PA}_{\mathrm{SD}}$ & 0.50 & 0.18 & & & & & & & $0.82 * * *$ & $-0.63 * * *$ & $-0.75 * * *$ \\
\hline $\mathrm{PA}_{\mathrm{RMSSD}}$ & 0.63 & 0.25 & & & & & & & & $-0.59 * * *$ & $-0.61 * * *$ \\
\hline PA\%DET & 64.22 & 18.32 & & & & & & & & & $0.80 * * *$ \\
\hline $\mathrm{PA} \% \mathrm{REC}$ & 50.38 & 17.25 & & & & & & & & & \\
\hline
\end{tabular}


Main effects of mean affect levels. All associations between mean levels of affect and the outcome variables were consistent with previous literature. Specifically, greater NA mean was associated with more depressive and somatic symptoms (see first rows in Tables 4 and 5). Higher PA mean was associated with fewer depressive symptoms and somatic symptoms (see eighth row in Tables 4 and 5).

Main effects of affect variability. Greater amounts of both PA and NA variability were associated with more depressive symptoms (NASD: $b=0.19, z=4.59, p<.001,95 \% \mathrm{CI}[0.11$, 0.27]; PA $\left.\mathrm{SD}_{\mathrm{SD}} b=0.31, z=7.64, p<.001,95 \% \mathrm{CI}[0.23,0.39]\right)$ and more somatic symptoms NA $_{\mathrm{SD}}: b=0.22, z=3.53, p<.001,95 \% \mathrm{CI}[0.10,0.34]$; $\mathrm{PA}_{\mathrm{SD}}: b=0.48, z=8.17, p<.001,95 \%$ CI $[0.36,0.59]$; see Tables 4 and 5 models 1 and 6). In other words, individuals who had more affect variability had worse health outcomes.

Main effects of affect predictability. Higher NA predictability was associated with fewer depressive symptoms $(b=-0.16, z=-3.25, p=.001,95 \%$ CI $[-0.26,-0.06])$ and fewer somatic symptoms $(b=-0.25, z=-3.31, p=.001,95 \%$ CI $[-0.39,-0.10]$; see Tables 4 and 5 model 3). PA predictability, on the other hand, was not associated with either depressive symptoms $(b=0.00, z=0.08, p=.938,95 \%$ CI $[-0.10 .0 .11])$ or somatic symptoms $(b=0.10, z$ $=1.40, p=.162,95 \%$ CI [-0.04, 0.25]; see Tables 4 and 5 model 8). In sum, individuals with more predictable NA, had better health outcomes while their PA predictability did not matter. 
Table 4

Mean Level, Variability, and Predictability Metrics of Affect Predicting Depressive Symptoms.

\begin{tabular}{|c|c|c|c|c|c|c|c|c|c|c|}
\hline & \multicolumn{10}{|c|}{ Models } \\
\hline & 1 & 2 & 3 & 4 & 5 & 6 & 7 & 8 & 9 & 10 \\
\hline $\mathrm{NA}_{\text {MEAN }}$ & $0.56 * * *$ & $0.61 * * *$ & $0.60 * * *$ & $0.60 * * *$ & $0.58 * * *$ & & & & & \\
\hline $\mathrm{NA}_{\mathrm{SD}}$ & $0.19 * * *$ & $0.32 * * *$ & $0.24 * * *$ & $0.21 * * *$ & $0.23 * * *$ & & & & & \\
\hline $\mathrm{NA}_{\text {MEAN }} * \mathrm{NA}_{\mathrm{SD}}$ & & $-0.69 * * *$ & $-0.66 * * *$ & $-0.55 * * *$ & $-0.51 * * *$ & & & & & \\
\hline NA\%DET & & & $-0.16^{* *}$ & $-0.21 * * *$ & $-0.22 * * *$ & & & & & \\
\hline NAMEAN $*$ NA\%DET & & & & 0.20 & 0.08 & & & & & \\
\hline $\mathrm{NA}_{\mathrm{SD}} * \mathrm{NA} \% \mathrm{DET}$ & & & & & $0.67 * *$ & & & & & \\
\hline $\mathrm{NA}_{\mathrm{MEAN}} * \mathrm{NA}_{\mathrm{SD}} * \mathrm{NA} \% \mathrm{DET}$ & & & & & -0.16 & & & & & \\
\hline PAMEAN & & & & & & $-0.48 * * *$ & $-0.48 * * *$ & $-0.48 * * *$ & $-0.48 * * *$ & $-0.47 * * *$ \\
\hline $\mathrm{PA}_{\mathrm{SD}}$ & & & & & & $0.31 * * *$ & $0.35 * * *$ & $0.35 * * *$ & $0.34 * * *$ & $0.37 * * *$ \\
\hline PAMEAN $* \mathrm{PA}_{\mathrm{SD}}$ & & & & & & & $0.33 * * *$ & $0.33 * * *$ & $0.29 * *$ & $0.30 * *$ \\
\hline PA\%DET & & & & & & & & 0.00 & -0.00 & -0.01 \\
\hline PAMEAN $^{*}$ PA $_{\% \mathrm{DET}}$ & & & & & & & & & -0.07 & -0.06 \\
\hline $\mathrm{PA}_{\mathrm{SD}} * \mathrm{PA} \% \mathrm{DET}$ & & & & & & & & & & $0.48 *$ \\
\hline PAMEAN $^{*} \mathrm{PA}_{\mathrm{SD}} * \mathrm{PA} \% \mathrm{DET}$ & & & & & & & & & & 0.39 \\
\hline Distress & 0.00 & -0.01 & -0.01 & -0.02 & -0.02 & $0.18 * * *$ & $0.18 * * *$ & $0.18 * * *$ & $0.18 * * *$ & $0.18 * * *$ \\
\hline Constant & $2.60 * * *$ & $2.66 * * *$ & $2.66^{* * *}$ & $2.67 * * *$ & $2.68 * * *$ & $2.37 * * *$ & $2.37 * * *$ & $2.37 * * *$ & $2.37 * * *$ & $2.38 * * *$ \\
\hline Observations & 1,301 & 1,301 & 1,301 & 1,301 & 1,301 & 1,300 & 1,300 & 1,300 & 1,300 & 1,300 \\
\hline
\end{tabular}

Note. ${ }^{*} p<.05, * * p<.01, * * * p<.001$. 
Table 5

Mean Level, Variability, and Predictability Metrics of Affect Predicting Somatic Symptoms.

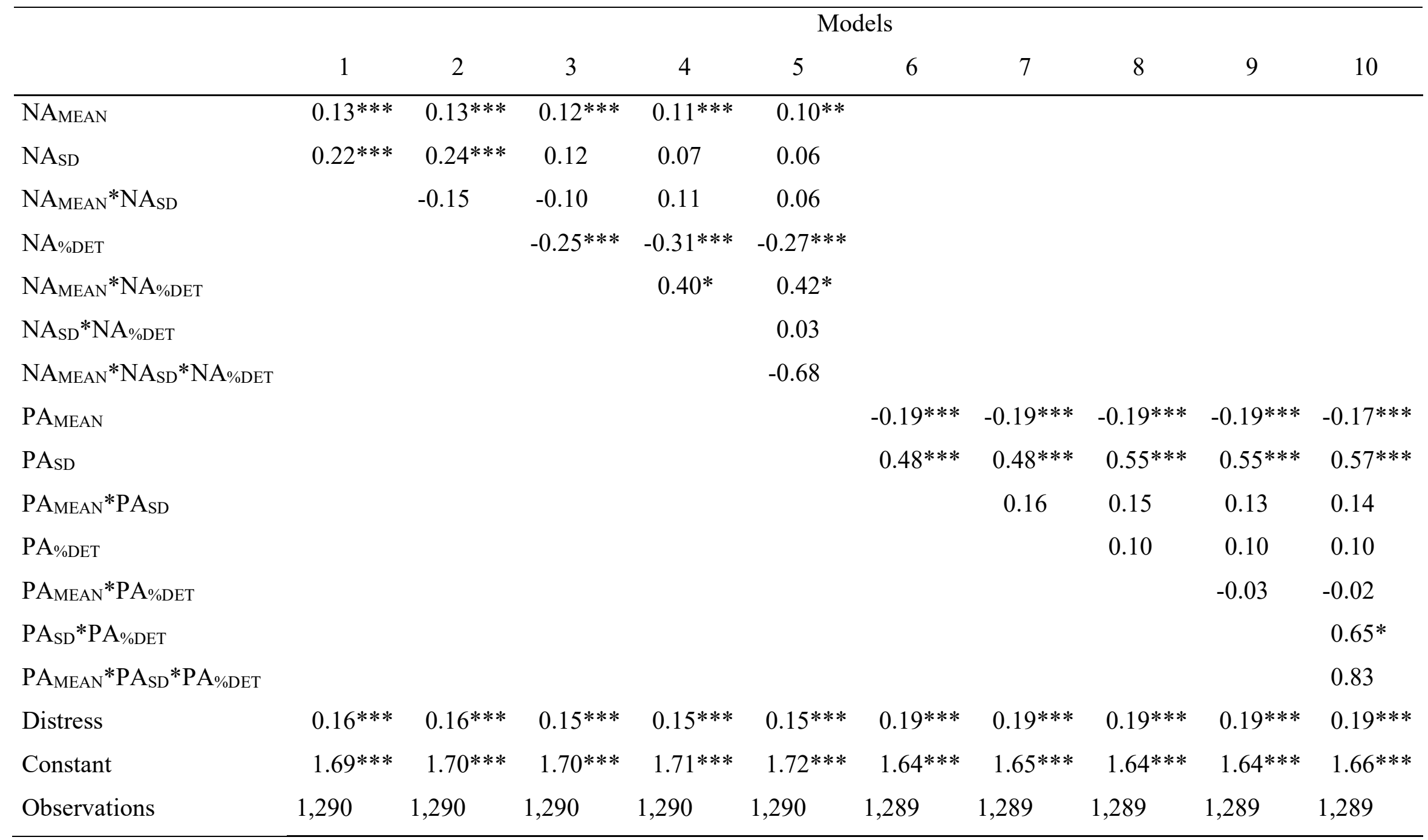

Note. ${ }^{*} p<.05, * * p<.01, * * * p<.001$. 
Interactions between variability and mean levels. NA variability interacted with NA mean to predict depressive symptoms $(b=-0.69, z=-10.63, p<.001,95 \%$ CI $[-0.81,-0.56])$ but not somatic symptoms $(b=-0.15, z=-1.58, p=.114,95 \%$ CI $[-0.34,0.04]$; see Tables 4 and 5 model 2). Specifically, at higher levels of NA mean, less NA variability was associated with greater depressive symptoms (see Figure 4). PA variability interacted with PA mean to predict depressive symptoms $(b=0.33, z=4.17, p<.001,95 \%$ CI $[0.18,0.49])$ but not somatic symptoms $(b=0.16, z=1.36, p=.175,95 \%$ CI $[-0.07,0.38]$; see Tables 4 and 5 model 7). At lower levels of PA mean, less PA variability was associated with more depressive symptoms (see Figure 5). This demonstrates that, as expected from previous characterizations of depressive symptoms (Watson, Clark, \& Carey, 1988), individuals with worse psychological health outcomes had consistently high NA and/or consistently low PA.

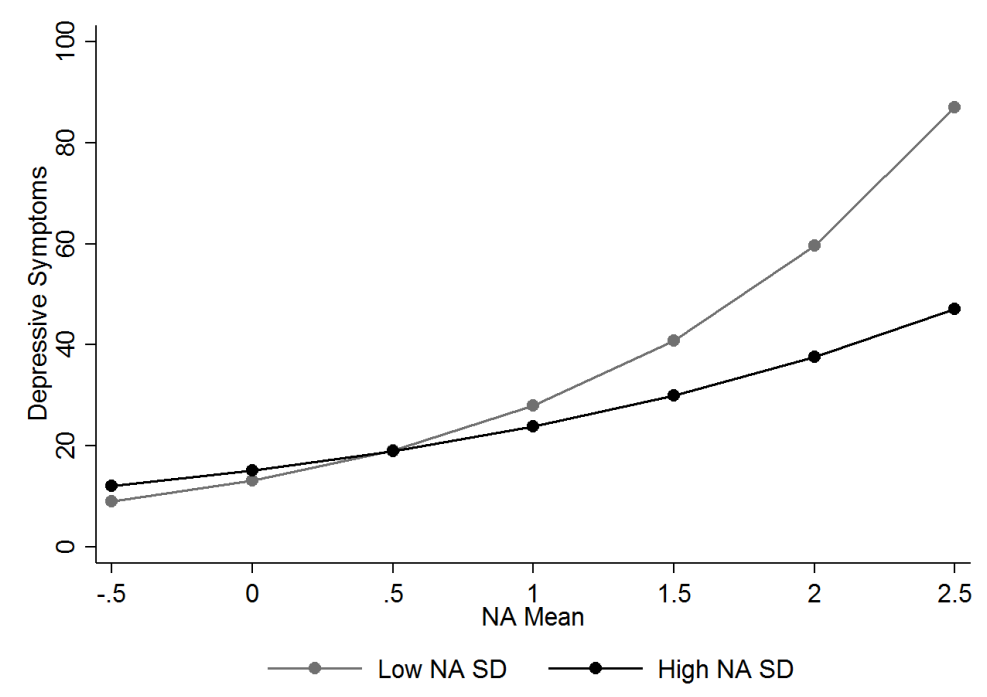

Figure 4. Interaction between NAMEAN and NASD predicting depressive symptoms. 


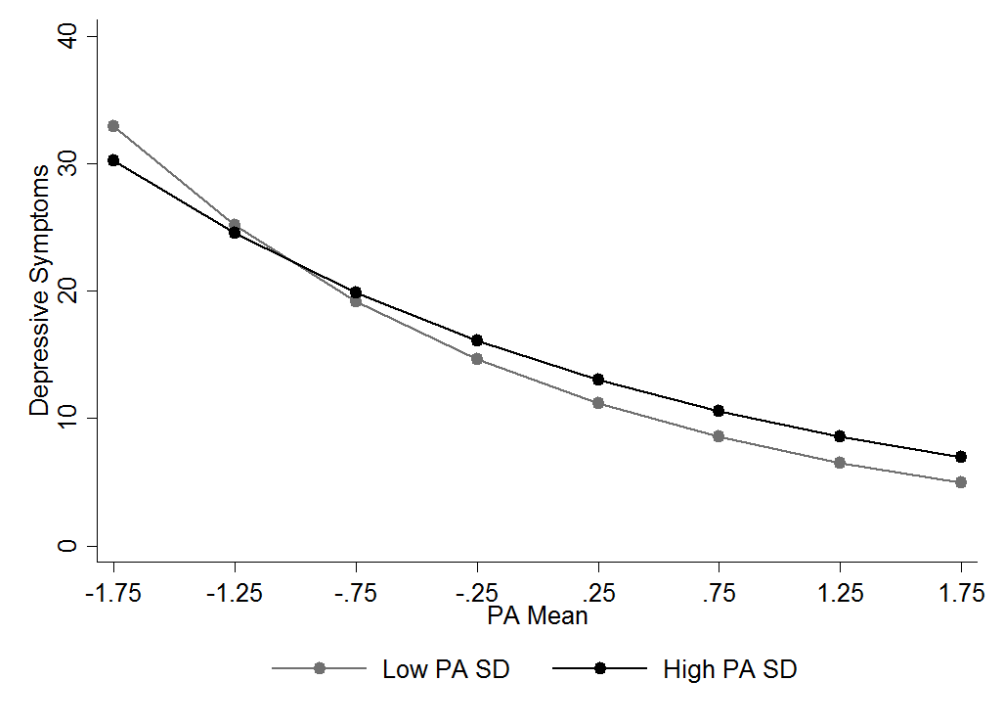

Figure 5. Interaction between $\mathrm{PA}_{\mathrm{MEAN}}$ and $\mathrm{PA}_{\mathrm{SD}}$ predicting depressive symptoms.

Interactions between predictability and mean levels. NA predictability interacted with NA mean to predict somatic symptoms $(b=0.40, z=2.55, p=.011,95 \% \mathrm{CI}[0.09,0.70])$ and, marginally, depressive symptoms $(b=0.20, z=1.96, p=.051,95 \%$ CI $[-0.00,0.39]$; see Tables 4 and 5 model 4). Specifically, at higher levels of NA mean, higher NA predictability was associated with marginally more depressive symptoms (see Figure 6a) and more somatic symptoms (see Figure 6b). PA variability did not interact with PA mean to predict depressive symptoms $(b=-0.07, z=-0.70, p=.483,95 \%$ CI $[-0.27,0.13])$ or somatic symptoms $(b=-0.03$, $z=-0.21, p=.835,95 \%$ CI $[-0.32,0.26]$; see Tables 4 and 5 model 9). In summary, NA predictability tended to be worse for health outcomes at higher levels of mean NA. This might represent someone who experiences a lot of day to day NA that occurs in regular patterns. In contrast, the effect of PA predictability did not change based on levels of PA mean. 

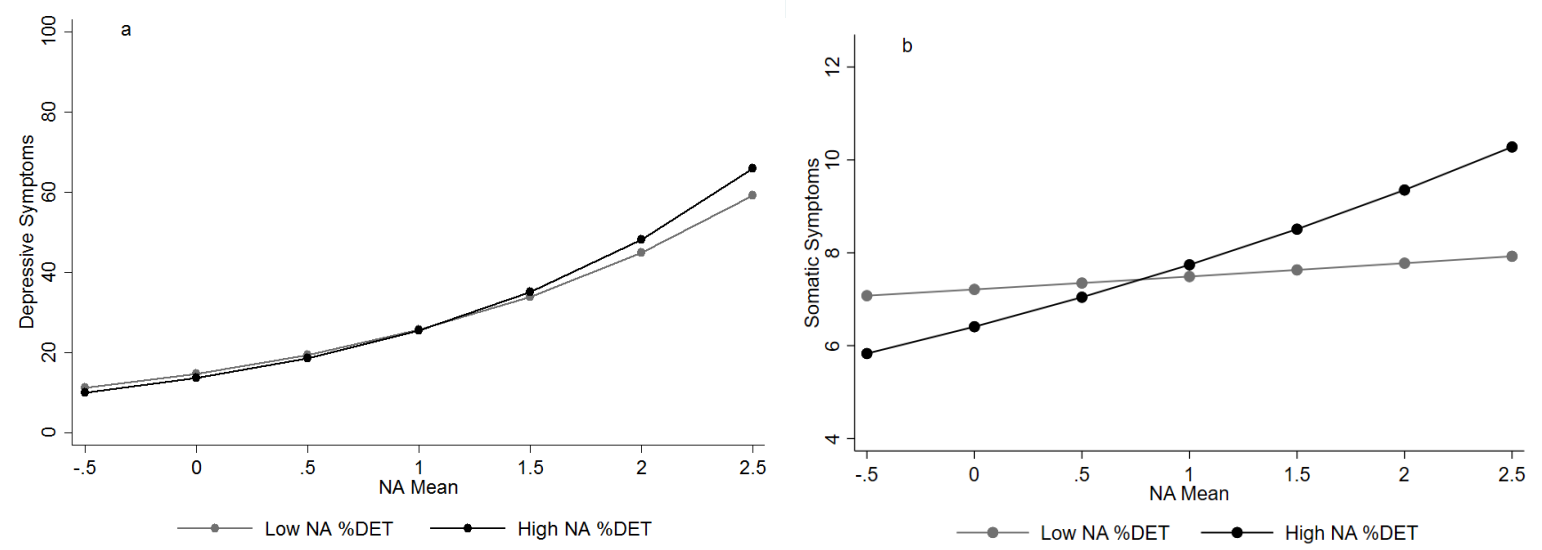

Figure 6. Interaction between NA $\mathrm{NEAN}_{\text {and }}$ NA\%DET predicting depressive and somatic symptoms.

Interactions between variability and predictability. NA predictability interacted with NA variability to predict depressive symptoms $(b=0.67, z=2.96, p=.003,95 \%$ CI $[0.23,1.11])$ but not somatic symptoms $(b=0.03, z=0.10, p=.918,95 \%$ CI $[-0.63,0.70]$; see Tables 4 and 5 model 5). At higher levels of NA variability, more NA predictability was associated more depressive symptoms (see Figure 7). PA predictability interacted with PA variability to predict depressive symptoms $(b=0.48, z=2.37, p=.018,95 \% \mathrm{CI}[0.08,0.87])$ and somatic symptoms $(b=0.65, z=2.24, p=.025,95 \%$ CI $[0.08,1.22]$; see Tables 4 and 5 model 10). At higher levels of PA variability, more PA predictability was associated with more depressive symptoms (see Figure 8a) and more somatic symptoms (see Figure 8b). As previously shown (see Tables 4 and 5 models 1 and 6), greater affect variability, whether it be NA or PA, was associated with worse outcomes. In these analyses of the interaction between variability and predictability, we see again that high levels of variability are detrimental and this is particularly true when affect is predictable. 


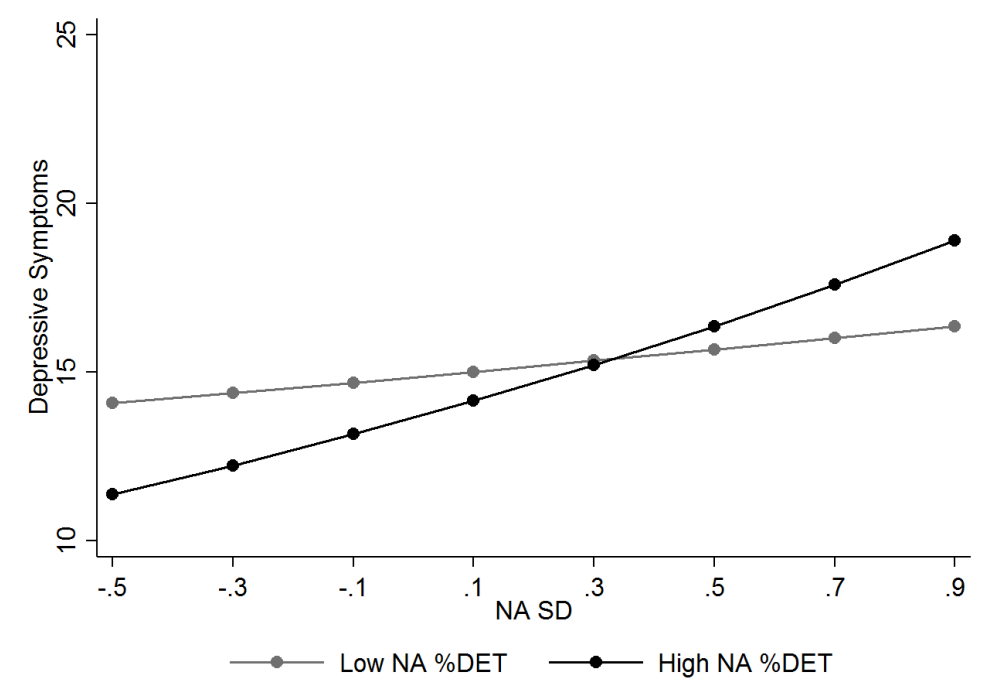

Figure 7. Interaction between NA\%DET and NA $\mathrm{SD}_{\text {p }}$ predicting depressive symptoms.
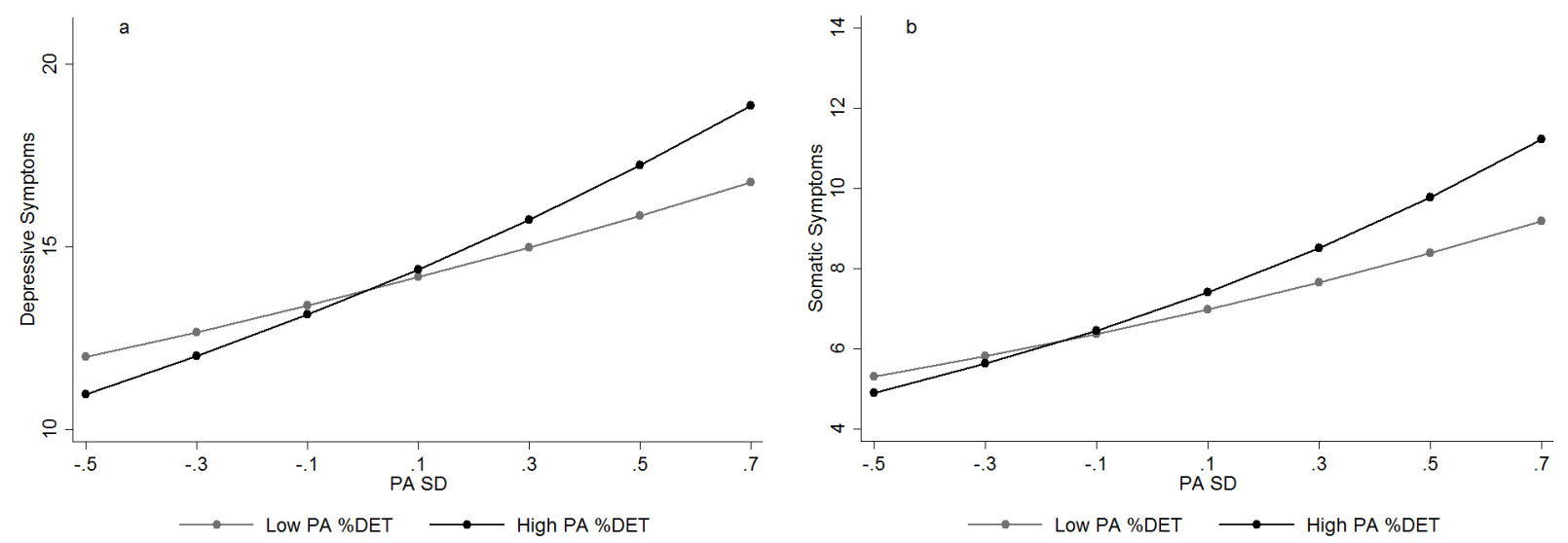

Figure 8. Interaction between $\mathrm{PA} \% \mathrm{DET}$ and $\mathrm{PA}_{\mathrm{SD}}$ predicting depressive symptoms and somatic symptoms.

Three-way interactions between variability, predictability, and mean. There were no three-way interactions between variability, predictability, and mean levels (all $p s>.05$; see Tables 4 and 5 models 5 and 10).

\section{Discussion}

This study shows for the first time that measures of affect predictability add important new information in regard to health outcomes. Critically, measures of predictability differed 
from measures of variability in their association with affect valence. For example, this study revealed that individuals with higher average NA had more variable but less predictable affect. Conversely, those with greater mean PA had less variable but more predictable affect. This implies that individuals high in NA generally have greater and more erratic fluctuations in affect compared to those who have low NA. Additionally, individuals high in PA generally have less intense and more stable fluctuations in affect compared to those who have low PA.

These factors of variability and predictability were not only associated with PA and NA differentially, but they also had different associations with health outcomes. In general, more variable NA and PA was associated with worse outcomes (i.e., more depressive and somatic symptoms), consistent with past studies on similar topics (Gruber et al., 2013; Hardy \& Segerstrom, 2016). On the other hand, our newly studied predictability metrics revealed that NA patterns that are more consistent were associated with better outcomes (i.e., fewer depressive and somatic symptoms) while PA predictability mattered less.

Drawing on the discrepancies found between predictability and variability, it is also informative to examine how these factors interact with mean levels of affect to predict those same health outcomes. Although variability generally resulted in less favorable outcomes, at higher levels of mean NA, lower NA variability actually became associated with worse outcomes. For example, individuals with higher mean levels of NA had more depressive symptoms when they had less variable NA. It is possible that less variation for those high in mean NA indicates they are constantly in a negative state, which amplifies the detrimental effects that NA has on health. In contrast, someone with high mean NA and with greater NA variability may at least get to have some "breaks" from their typical high levels of negativity, which ultimately leads to more favorable health outcomes. For PA, variability had a similar 
effect whereby at lower levels of mean PA, less variability was associated with worse outcomes. For these individuals, less variability means that they are consistently low in positivity. In these ways, less variability has similar effects but depends on the valence and mean level of affect.

More NA predictability was generally associated with better outcomes. However, higher levels of NA predictability were associated with worse outcomes for those with high levels of NA. High predictability of NA for those already high in NA could signify that a person is "stuck" in a negative situation that they are continually experiencing. Someone who feels poorly now and expects to continue feeling poorly in the future will likely exhibit the least desirable outcomes, consistent with dynamic systems approaches to resilience (Pincus \& Metten, 2010). PA predictability, on the other hand, did not have a main effect on the health outcomes nor did it interact with mean PA. However, the interaction between variability and predictability for both NA and PA was significant such that at high levels of variability, high predictability was worse for health. In sum, consistent NA predictability was generally better for health, except when NA mean was high. In addition, consistent PA and NA predictability were each worse for health when variability levels were high.

Based on these findings, researchers should consider using predictability metrics in addition to variability metrics, and this is especially true for NA. Furthermore, using interaction terms will provide opportunities to see how variability and predictability play divergent roles for different levels of mean affect. When we consider the nature of repeated patterns of affect (i.e., RQA metrics) in addition to measures of variability, the conclusions drawn about how affect changes wellness outcomes are altered. Assessing variability on its own is important, but additionally assessing predictability provides a clearer and more interesting picture about how fluctuations in affect influence mental and physical health. 
Further, these findings demonstrate the importance of using multiple metrics of variability to test the robustness of findings. For example, standard deviation and RMSSD were very highly correlated $(r \mathrm{~s}>.80$, see Table 3$)$ demonstrating that they are likely tapping the same construct, namely, affect variability. While these metrics are mathematically similar, they each independently reveal a more nuanced understanding of affect variability by providing conceptually different information about how affect changes over time. RMSSD determines changes in affect from one time point to the next, which illuminates how the temporal ordering of affective events influences variability. On the other hand, standard deviation represents the magnitude of change in affect but ignores the sequential order of events. On their own, they each provide only one piece of the interconnected puzzle, but when taken together we can understand how the magnitude and sequential changes of affect influence health.

There are a number of limitations in this work. First, we are unable to make causal conclusions about how affect is related to health, which leaves open the possibility of reverse causation. More variability could conceivably lead to higher levels of depressive symptoms, but it is also possible that having high levels of depressive symptoms could lead to more variable affect. Similarly, high somatic symptoms could have led to changes in affect variability and predictability. As in other observational studies on affect, our study design does not allow us to answer these types of directional questions. Nevertheless, this study adds substantially to the affect literature by demonstrating that predictability plays at least some role in the affect-health association. A second limitation is the relatively short time frame of data collection. It is possible that adding an additional week of data could have helped us more accurately assess predictability. However, data collection during a third week would add additional strain on participant adherence. Future research may consider examining different time spans of data 
collection. Additionally, it must be acknowledged that variability and predictability are highly correlated. This high correlation partially accounts for why there were sometimes no significant effects of PA predictability. However, these metrics are not perfectly correlated, which allows the RQA metrics to further differentiate certain cases and provide additional information. Another limitation is that our assessment methodology only focused on self-reported affect and did not take into account the variety of factors (e.g., stress, daily experiences) that may have contributed to fluctuations in affect. In future studies, it may be informative to monitor daily activities and contexts in order to paint a more refined picture of what drives these affective changes. Finally, the results of our study are not generalizable to the population at large because of our limited study sample. The participants were primarily Caucasian undergraduates, so our conclusions only apply to these types of individuals. However, these methods may be extended to other populations and so future research may address this gap.

There are important conclusions about the divergent impacts of variability and predictability that deserve consideration. In general, it was found that the most desirable outcomes stemmed from those who had high PA, low NA, low variability, and high predictability. PA did not interact with variability or predictability (high PA was good in all scenarios), but there were some interesting findings in regard to NA. The results indicate that for individuals high in NA mean, it is good to have high variability (possibly because one gets "breaks" from the negativity) and it is bad to have high predictability (possibly because the negativity is unchangeable). As demonstrated by these findings, the additional predictability measures add explanatory depth to how the dynamics of affect are associated with health.

These studies are the first to demonstrate how RQA metrics (\%DET and \%REC) can add interesting new information about the association between affective experiences and health 
outcomes by elucidating temporal patterns that are often overlooked when relying only on variability or mean levels. When assessing psychosomatic connections, the vast majority of studies rely on indicators of mean affect (Pressman \& Cohen, 2005). Our findings should implore future researchers to also consider the role of predictability as well as encourage the growing interest in affect variability and health. Affect unfolds over time, so the consideration of temporal patterns is critical in order to capture the dynamic nature of affective experiences (Jenkins, 2017). 


\section{References}

Anderson, N. C., Bischof, W. F., Laidlaw, K. E. W., Risko, E. F., \& Kingstone, A. (2013). Recurrence quantification analysis of eye movements. Behavior Research Methods, 45, 842-56. https://doi.org/10.3758/s13428-012-0299-5

Barrett, L. F., \& Russell, J. A. (1999). The structure of current affect: Controversies and emerging consensus. Current Directions in Psychological Science, 8, 10-14. https://doi.org/10.1111/1467-8721.00003

Chida, Y., \& Steptoe, A. (2008). Positive psychological well-being and mortality: A quantitative review of prospective observational studies. Psychosomatic Medicine, 70, 741-56. https://doi.org/10.1097/PSY.0b013e31818105ba

Ebner-Priemer, U. W., Eid, M., Kleindienst, N., Stabenow, S., \& Trull, T. J. (2009). Analytic strategies for understanding affective (in)stability and other dynamic processes in psychopathology. Journal of Abnormal Psychology, 118, 195-202. https://doi.org/10.1037/a0014868

Eid, M., \& Diener, E. (1999). Intraindividual variability in affect: Reliability, validity, and personality correlates. Journal of Personality and Social Psychology, 76, 662-676. https://doi.org/10.1037/0022-3514.76.4.662

Faul, F., Erdfelder, E., Buchner, A., \& Lang, A. G. (2009). Statistical power analyses using G*Power 3.1: Tests for correlation and regression analyses. Behavioral Research Methods, $41,1149-1160$.

Gruber, J., Kogan, A., Quoidbach, J., \& Mauss, I. B. (2013). Happiness is best kept stable: Positive emotion variability is associated with poorer psychological health. Emotion, 13, 16. https://doi.org/10.1037/a0030262 
Hardy, J., \& Segerstrom, S. C. (2016). Intra-individual variability and psychological flexibility: Affect and health in a National US sample. Journal of Research in Personality. https://doi.org/10.1016/j.jrp.2016.04.002

Houben, M., Van Den Noortgate, W., \& Kuppens, P. (2015). The relation between short-term emotion dynamics and psychological well-being: A meta-analysis. Psychological Bulletin, 141, 901-930. https://doi.org/10.1037/a0038822

Human, L. J., Whillans, A. V., Hoppmann, C. A., Klumb, P., Dickerson, S. S., \& Dunn, E. W. (2015). Finding the middle ground: Curvilinear associations between positive affect variability and daily cortisol profiles. Emotion, 15, 705-720. https://doi.org/10.1037/emo0000071

Jenkins, B. N. (2017). Affect variability is constantly important: Implications for health. Jenkins, B. N., Granger, D. A., Roemer, R. J., Martinez, A., Torres, T. K., \& Fortier, M. A. (2018). Emotion regulation and positive affect in the context of salivary alpha-amylase response to pain in children with cancer. Pediatric Blood \& Cancer, e26973. https://doi.org/10.1002/pbc.26973

Jenkins, B. N., Hunter, J. F., Cross, M. P., Acevedo, A. M., \& Pressman, S. D. (2018). When is affect variability bad for health? The association between affect variability and immune response to the influenza vaccination. Journal of Psychosomatic Research, 104, 41-47. https://doi.org/10.1016/j.jpsychores.2017.11.002

Kuppens, P., Allen, N. B., \& Sheeber, L. B. (2010). Emotional inertia and psychological maladjustment. Psychological Science, 21, 984-991. https://doi.org/10.1177/0956797610372634

Main, A., Paxton, A., \& Dale, R. (2016). An exploratory analysis of emotion dynamics between 
mothers and adolescents during conflict discussions. Emotion, 16, 913-928. https://doi.org/10.1037/emo0000180

Peeters, F., Berkhof, J., Delespaul, P., Rottenberg, J., \& Nicolson, N. A. (2006). Diurnal mood variation in major depressive disorder. Emotion, 6, 383-391. https://doi.org/10.1037/15283542.6.3.383

Pincus, D., \& Metten, A. (2010). Nonlinear dynamics in biopsychosocial resilience. Nonlinear Dynamics, Psychology, and Life Sciences, 14, 353-380.

Pressman, S. D., \& Cohen, S. (2005). Does positive affect influence health? Psychological Bulletin, 131, 925-71. https://doi.org/10.1037/0033-2909.131.6.925

Pressman, S. D., Jenkins, B. N., Kraft-Feil, T. L., Rasmussen, H., \& Scheier, M. F. (2017). The whole is not the sum of its parts: Specific types of positive affect influence sleep differentially. Emotion, 17. https://doi.org/10.1037/emo0000256

Pressman, S. D., Jenkins, B. N., \& Moskowitz, J. T. (2019). Positive affect and health: What do we know and where next should we go ?, 1-24.

Radloff, L. S. (1977). The CES-D Scale: A self-report depression scale for research in the general population. Applied Psychological Measurement, 1, 385-401.

Ram, N., \& Gerstorf, D. (2009). Time-structured and net intraindividual variability: Tools for examining the development of dynamic characteristics and processes. Psychology and Aging, 24, 778-91. https://doi.org/10.1037/a0017915

Richardson, M. J., Dale, R., \& Marsh, K. L. (2014). Complex dynamical systems in social and personality psychology. In Handbook of research methods in social and personality psychology (pp. 253-282).

Riley, M. A., \& Clark, S. (2003). Recurrence analysis of human postural sway during the sensory 
organization test. Neuroscience Letters, 342, 45-48. https://doi.org/10.1016/S03043940(03)00229-5

Röcke, C., \& Brose, A. (2013). Intraindividual variability and stability of affect and well-being. GeroPsych, 26, 185-199. https://doi.org/10.1024/1662-9647/a000094

Röcke, C., Li, S.-C., \& Smith, J. (2009). Intraindividual variability in positive and negative affect over 45 days: Do older adults fluctuate less than young adults? Psychology and Aging, 24, 863-878. https://doi.org/10.1037/a0016276

StataCorp. (2017). Stata Statistical Software: Release 15. College Station, TX: StataCorp LLC.

Steiger, J. H. (2004). Beyond the F test: Effect size confidence intervals and tests of close fit in the analysis of variance and contrast analysis. Psychological Methods, 9, 164-182. https://doi.org/10.1037/1082-989X.9.2.164

Watson, D., Clark, L. A., \& Carey, G. (1988). Positive and negative affectivity and their relation to anxiety and depressive disorders. Journal of Abnormal Psychology, 97, 346-353. https://doi.org/10.1037/0021-843X.97.3.346 


\section{Supplemental Material A}

1. Create dataset: Create an excel data set with only variables for RQA and ID (see example Figure 1). The RQA software requires a minimum of 10 data points.

Figure 1. Dataset with only ID and variables 1 (V1) through 12 (V12)

\begin{tabular}{|c|c|c|c|c|c|c|c|}
\hline 4 & A & B & C & D & $\mathrm{E}$ & $\mathrm{F}$ & G \\
\hline 1 & ID & V1 & V2 & V3 & V4 & V5 & V6 \\
\hline 2 & 1 & 2 & 2 & 5 & 3 & 4 & 4 \\
\hline 3 & 2 & 1 & 2 & 5 & 4 & 2 & 2 \\
\hline 4 & 3 & 3 & 3 & 3 & 2 & & 5 \\
\hline 5 & 4 & 5 & & 4 & 4 & 3 & 2 \\
\hline 6 & 5 & 5 & 4 & 2 & 2 & 2 & 3 \\
\hline 7 & & & & & & & \\
\hline 8 & & & & & & & \\
\hline
\end{tabular}

2. Set up macro: Under the "View" tab in excel, click "Macros" and then "View Macros" (see Figure 2). Paste "savemyrowsas text" into the macro name and click "Create" (see Figure 3).

Figure 2. Location of Macros button.

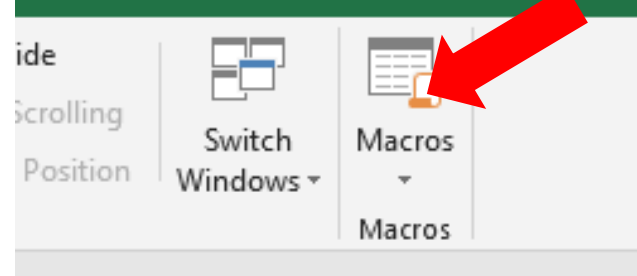

Figure 3. Naming and creating your macro.

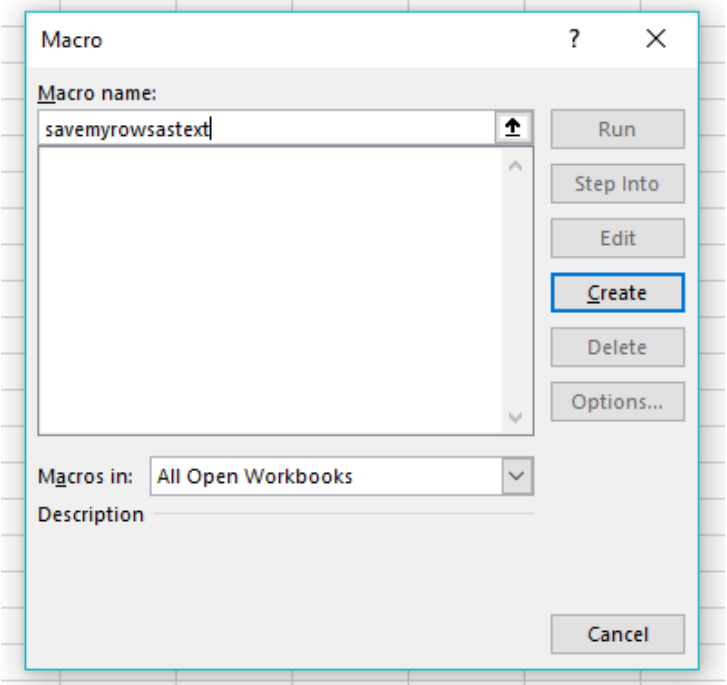


3. Create a separate text file for each participant: Paste code 1 (see Code 1 below) in between "Sub savemyrowsastext ()" and "End Sub" (see Figure 4). The yellow highlighted code is where your files will be saved. Alter this code to match the folder on your computer where you would like to save your data. The green highlighted code is which cells have the data. For example, "cell. Offset $(0,1)$ " indicates that the first datapoint is in the second column. This code goes up to 12 columns but if you have more data, you can just add " \& vbNewLine \& cell. Offset $(0,12)$ " altering the number just before the close parentheses. When you save your excel file, make sure to save your excel file as Macro enabled.

\section{Code 1:}

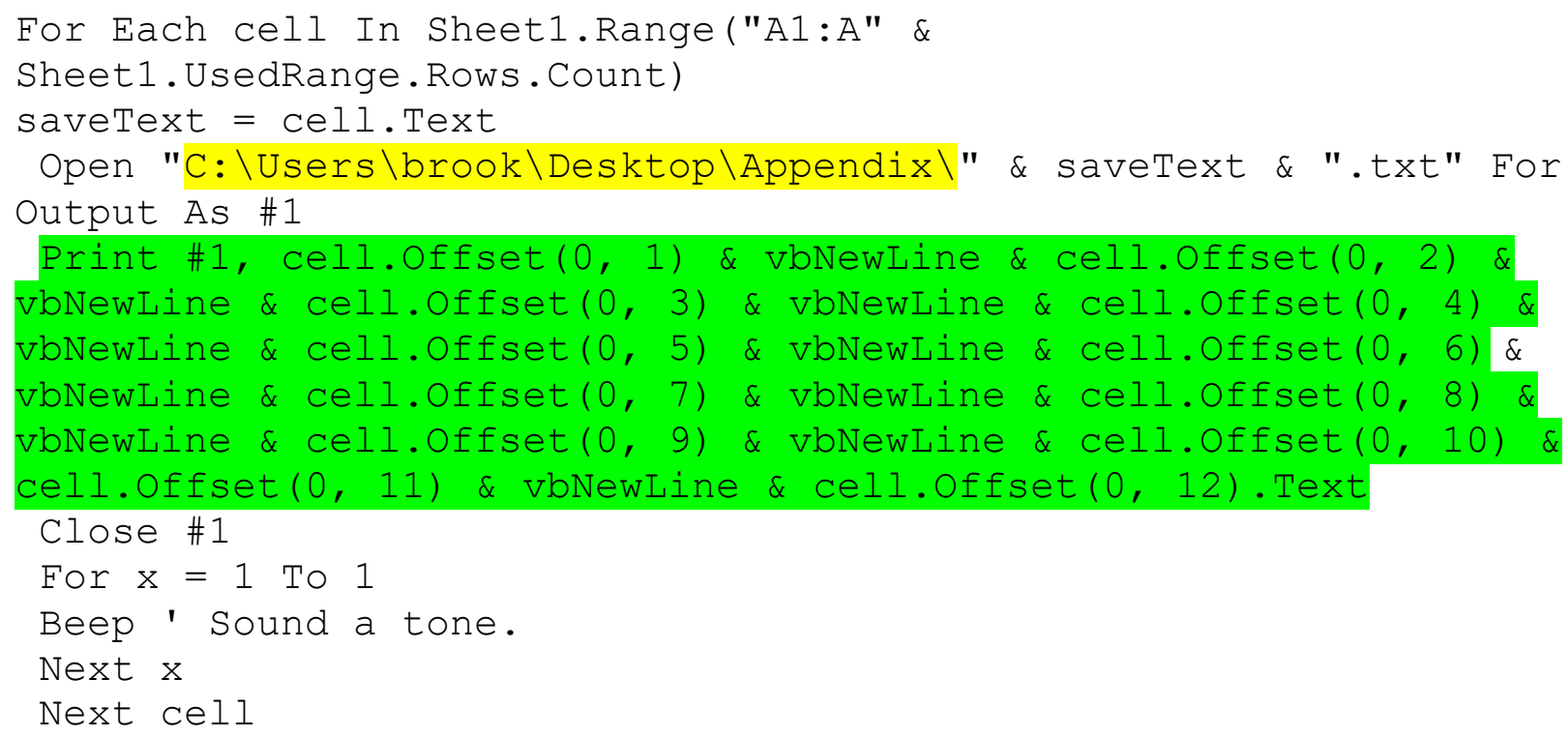

Figure 4. Screenshot of code in excel macro.

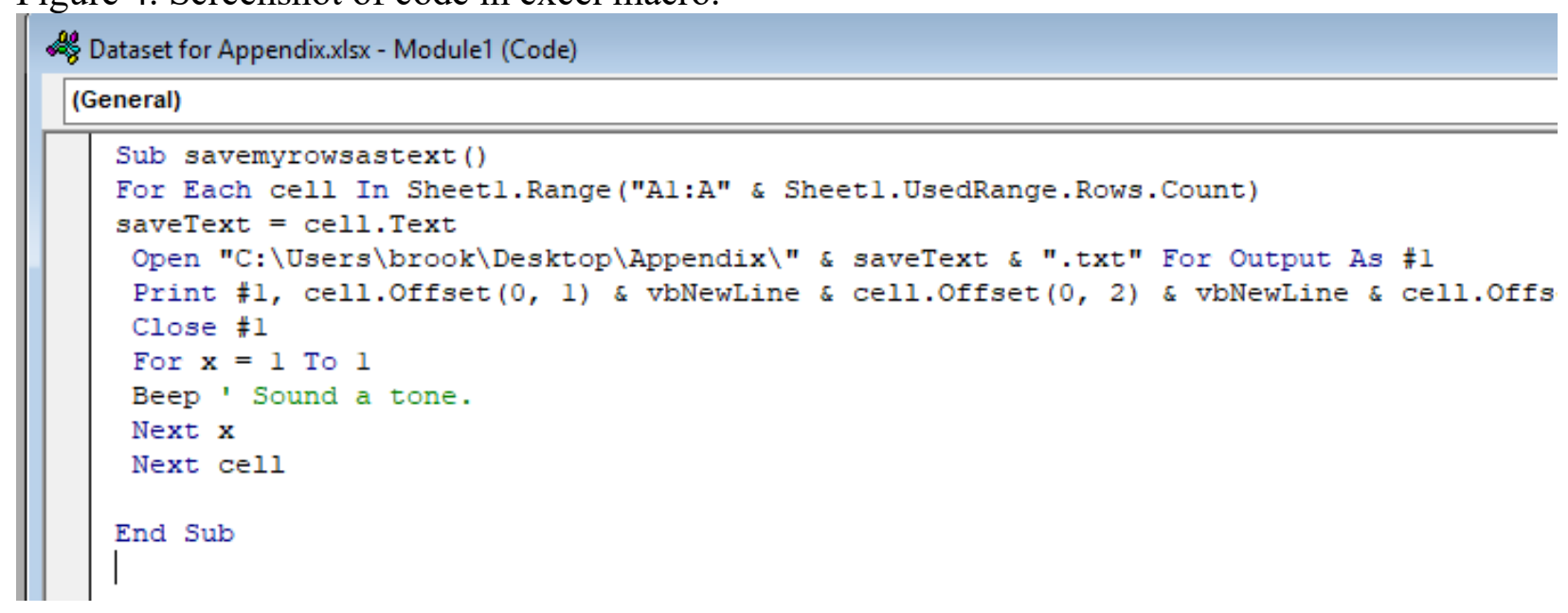


4. Run macro: Highlight all code and click the green arrow to run the code (see Figure 5). You can check that this code worked by going to the folder where your text files are saved (see Figure 6). Open a few of them to ensure that the data in the text file matches the data from your excel file (see Figure 7).

Figure 5. Run button.

plications - Dataset for Appendix.

Format Debug Run Iools

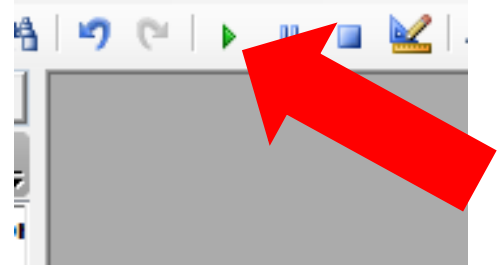

Figure 6. Folder with text files.

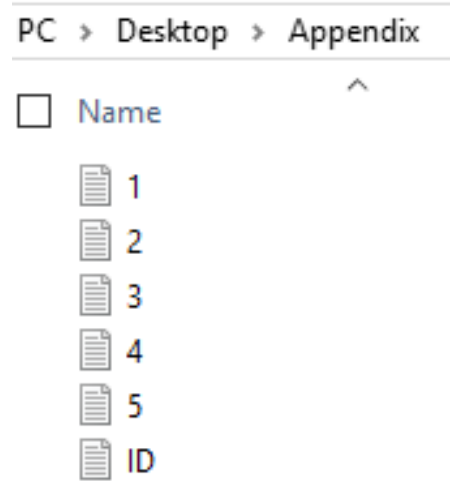

Figure 7. Text file of participant 4 data.

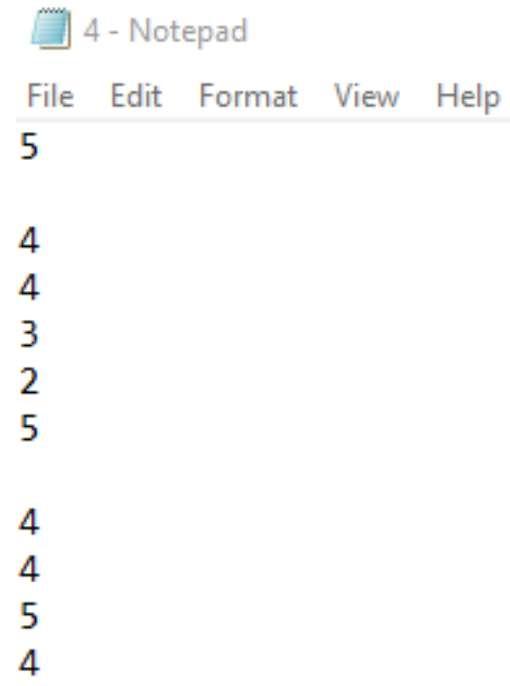


5. Create one file with all ID numbers listed: Use code 2 (see Code 2 below; see Figure 8 ) to create a text file that has all the ID numbers. The blue highlighted text is the cells to pull data from. In this example, we are pulling from the first column rows 2 through 6. You can adjust this code as needed. The yellow highlighted code is where the file will be saved. Notice that "batchlist" has been added to the end of this code; this was done to name the file "batchlist." Go to the folder where the text file is saved and open the batchlist.txt file to make sure all your ID numbers with ".txt." at the end are saved.

\section{Code 2:}

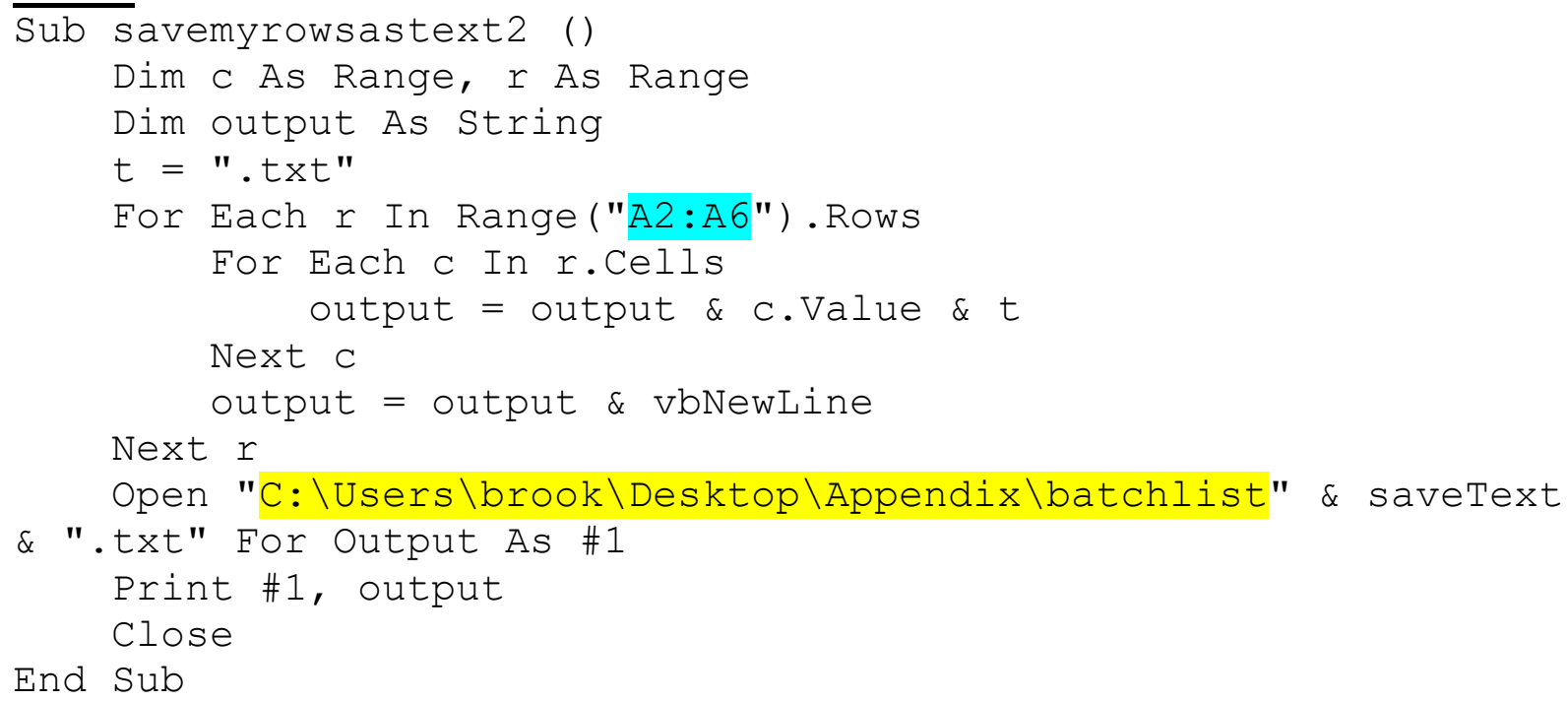

Figure 8 .

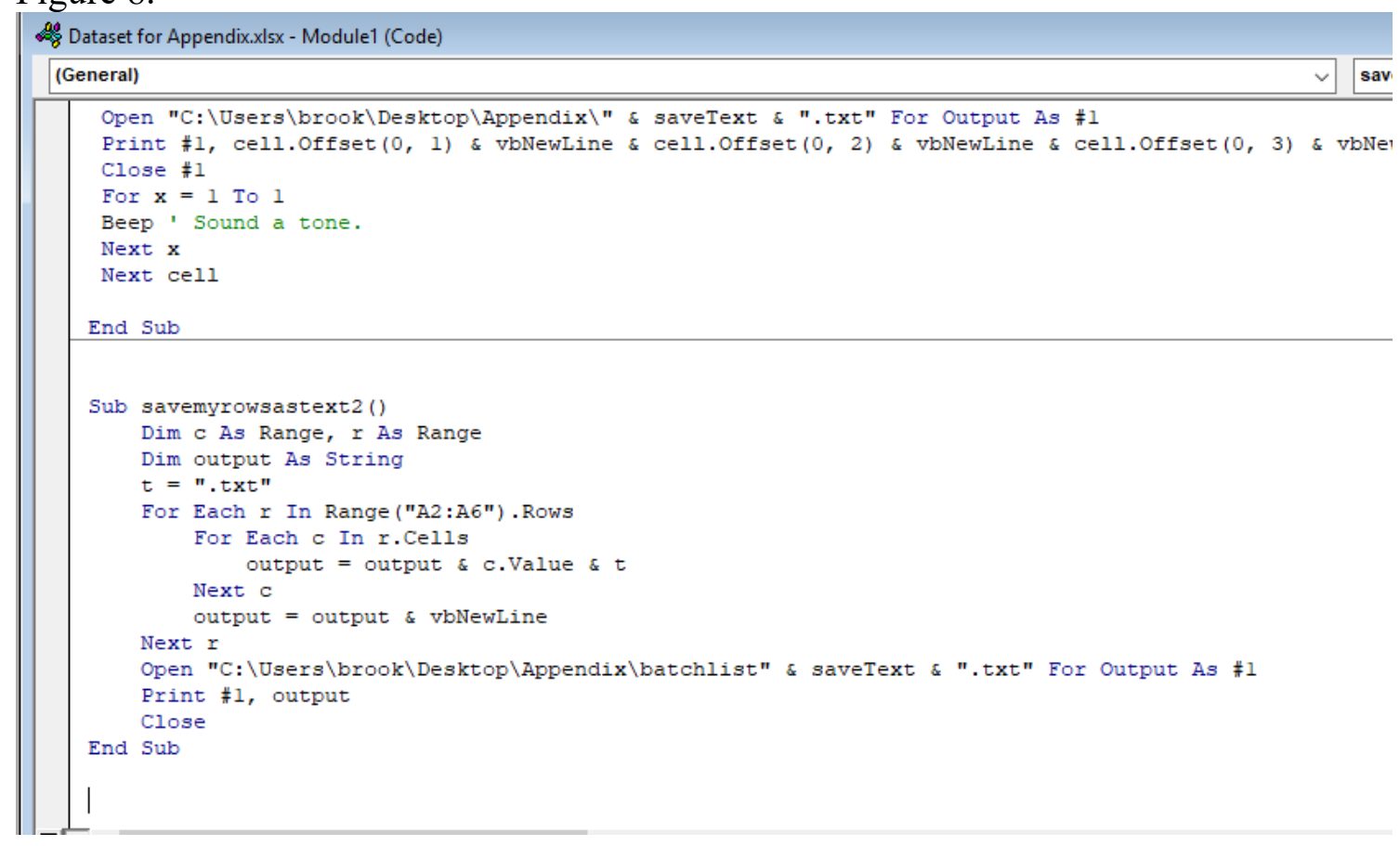


6. Access Matlab runtime compiler: Matlab runtime compiler is necessary to run the RQA software. Download Matlab runtime compiler from here:

https://www.mathworks.com/products/compiler/matlab-runtime.html?refresh=true\#\#

You will need 2017b MATLAB Runtime Component Compiler 64-bit

7. Dowload RQA program: Go to https://github.com/xkiwilabs/RQA-and-CRQA-GUIApplication and then click on "Compiled Applications." Download and save the rqa.exe program in the same file as your batchlist and each separate participant text file (see Figure 9).

Figure 9. RQA program, batchlist, and individual participant text files all saved in one folder.

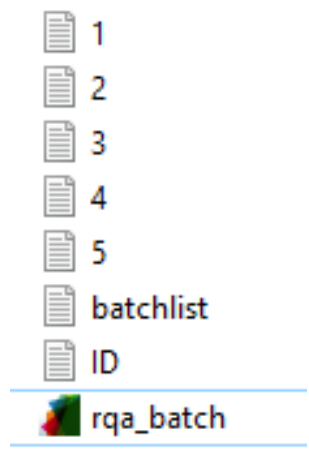

8. Run RQA: Remember that the Matlab runtime compiler is needed on your desktop in order to run the RQA program. Open the RQA program. Check the boxes "Categorical Recurrence" and "Batch." Then, click "Open Batch File" and select your batchlist file created in step 5 (see Figure 10). Click "Run."

Figure 10. RQA program with batchlist loaded and categorical recurrence and batch checked.

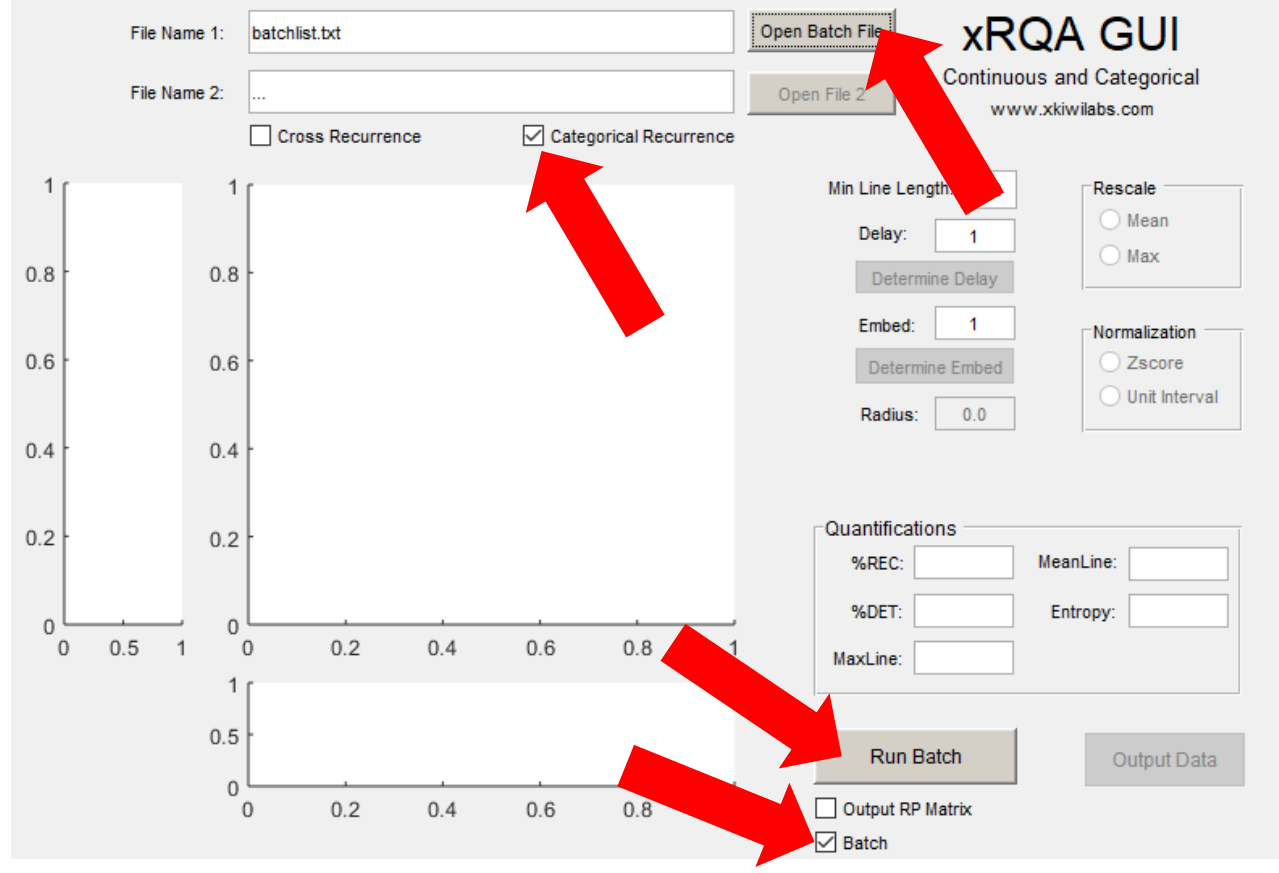


9. View RQA statistics: The RQA program will create an excel file with all the statistics it computed (see Figure 11). You can then merge this excel file with your other data to use the RQA statistics in your analyses.

Figure 11. RQA Stats excel file.

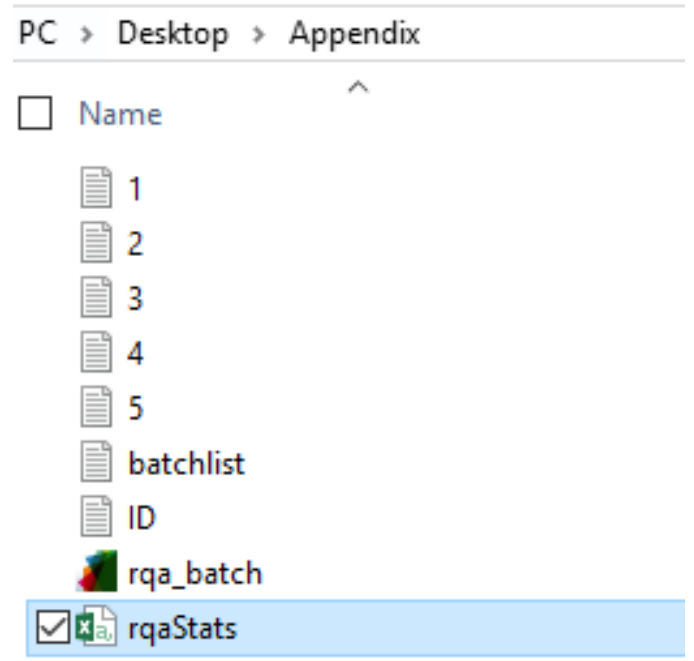

Common issues:

Make sure labels on participant text files (created in step 4) match the batchlist (created in step 5). One way to ensure this is by making sure ID numbers have no decimals. The excel macro code (described in step 4) uses the ID number to name the individual files but the RQA batchlist will not name the files with decimals (if they are there).

Make sure missing data are blank spaces. Sometimes excel uses \#NULL! to indicate missing data. Replace \#NULL! with [blank].

The ID variable in the RQA stats excel file will now have .txt in the name. To get the ID number without the .txt, you can use this formula (A2 is the cell you want to grab the number from): $=$ SUMPRODUCT(MID(0\&A2,LARGE(INDEX(ISNUMBER(--MID(A2,ROW(\$1:\$25),1))* $\left.\operatorname{ROW}(\$ 1: \$ 25), 0), \operatorname{ROW}(\$ 1: \$ 25))+1,1) * 10^{\wedge} \operatorname{ROW}(\$ 1: \$ 25) / 10\right)$

After using this formula, copy the column as numbers only (without the formula). 


\section{Supplemental Material B}

\section{Method}

We conducted the same analyses and models as reported in the manuscript but replaced standard deviation with emotional inertia. Emotional inertia was created for both NA and PA by calculating the autocorrelation with a lag of one similar to other work in this area (e.g., Kuppens, Allen, \& Sheeber, 2010). This resulted in the variables NAINERTIA and PAINERTIA.

\section{Results}

Descriptive statistics. Table 1 presents the means and standard deviations of the affect metrics as well as their associations. NAINERTIA and PA other measures of variability (standard deviation and RMSSD) as compared to the high correlations between standard deviation and $\operatorname{RMSSD}(r \mathrm{~s}>.80$ for each valence type).

Additionally, NAINERTIA and PAINERTIA were not highly correlated with measures of predictability (\%DET and \%REC). 
Table 1

Mean, Standard Deviation, and Pearson's Correlation of Affect Metrics.

\begin{tabular}{|c|c|c|c|c|c|c|c|c|c|c|c|c|c|}
\hline & Mean & SD & $\mathrm{NA}_{\mathrm{SD}}$ & $\mathrm{NA}_{\mathrm{RMSSD}}$ & $\mathrm{NA}_{\text {INERTIA }}$ & NA\%DET & $\mathrm{NA}_{\% \mathrm{REC}}$ & PA $_{\text {MEAN }}$ & $\mathrm{PA}_{\mathrm{SD}}$ & $\mathrm{PA}_{\mathrm{RMSSD}}$ & PA $_{\text {INERTIA }}$ & PA\%DET & $\mathrm{PA}_{\% \mathrm{REC}}$ \\
\hline $\mathrm{NA}_{\mathrm{MEAN}}$ & 1.67 & 0.47 & $0.64 * * *$ & $0.57 * * *$ & $0.08 * *$ & $-0.52 * * *$ & $-0.59 * * *$ & $-0.36 * * *$ & $0.17 * * *$ & $0.16^{* * *}$ & -0.04 & $-0.12 * * *$ & $-0.12 * * *$ \\
\hline $\mathrm{NA}_{\mathrm{SD}}$ & 0.41 & 0.22 & & $0.90 * * *$ & $0.13 * * *$ & $-0.66 * * *$ & $-0.76 * * *$ & $-0.24 * * *$ & $0.44 * * *$ & $0.39 * * *$ & 0.00 & $-0.30 * * *$ & $-0.32 * * *$ \\
\hline NARMSSD & 0.52 & 0.28 & & & $-0.22 * * *$ & $-0.65 * * *$ & $-0.69 * * *$ & $-0.21 * * *$ & $0.40 * * *$ & $0.44 * * *$ & $-0.11 * * *$ & $-0.27 * * *$ & $-0.29 * * *$ \\
\hline NA ${ }_{\text {INERTIA }}$ & 0.08 & 0.33 & & & & 0.00 & $-0.13 * * *$ & -0.05 & 0.05 & $-0.10 * * *$ & $0.24 * * *$ & -0.04 & $-0.07 * *$ \\
\hline NA\%DET & 71.67 & 19.03 & & & & & $0.84 * * *$ & $0.26 * * *$ & $-0.29 * * *$ & $-0.29 * * *$ & 0.05 & $-0.22 * * *$ & $-0.23 * * *$ \\
\hline $\mathrm{NA}_{\% \mathrm{REC}}$ & 58.36 & 21.11 & & & & & & $0.29 * * *$ & $-0.34 * * *$ & $-0.31 * * *$ & 0.01 & $0.25^{* * *}$ & $0.28 * * *$ \\
\hline PA $_{\text {MEAN }}$ & 3.00 & 0.50 & & & & & & & $-0.12 * * *$ & $-0.10 * * *$ & 0.01 & $0.06 * * *$ & $0.09 * * *$ \\
\hline $\mathrm{PA}_{\mathrm{SD}}$ & 0.50 & 0.18 & & & & & & & & $0.82 * * *$ & $0.17 * * *$ & $-0.63 * * *$ & $-0.75 * * *$ \\
\hline $\mathrm{PA}_{\mathrm{RMSSD}}$ & 0.63 & 0.25 & & & & & & & & & $-0.35 * * *$ & $-0.59 * * *$ & $-0.61 * * *$ \\
\hline PA $_{\text {INERTIA }}$ & 0.09 & 0.34 & & & & & & & & & & $-0.06^{*}$ & $-0.15 * * *$ \\
\hline $\mathrm{PA}_{\% \mathrm{DET}}$ & 64.22 & 18.32 & & & & & & & & & & & $0.80 * * *$ \\
\hline $\mathrm{PA}_{\% \mathrm{REC}}$ & 50.38 & 17.25 & & & & & & & & & & & \\
\hline
\end{tabular}

Note. $\mathrm{SD}=$ Standard Deviation. RMSSD $=$ root mean squared successive difference. $\% \mathrm{DET}=$ percent determinism. \%REC $=$ percent recurrence. ${ }^{*} p<.05,{ }^{* *} p<.01,{ }^{* * *} p<.001$ 
Negative affect. Mean, variability, and predictability of NA resulted in the same pattern of effects when inertia as opposed to standard deviation was used as a variability metric to predict depressive and somatic symptoms (see Tables 2 and 3 models 1 through 5). There were only two exceptions. First, the NAMEAN*NA\%DET interaction effect predicting depressive symptoms went from marginal in the manuscript to significant in these supplemental analyses (see highlighted coefficients in Table 2 models 4 and 5). Second, the main effect of NAINERTIA predicting somatic symptoms became non-significant (as compared to the main effect of NASD) in models 1 and 2 of these supplemental analyses (see highlighted coefficients in Table 3 models 1 and 2).

Positive affect. In the PA models predicting depressive symptoms, PAINERTIA had different effects on our outcomes of interest and also impacted other terms in the analyses (see highlighted coefficients in Table 2 models 6 through 10). For example, PAINERTIA had a negative effect on depressive symptoms as compared to the positive effect PA $A_{S D}$ had. Specifically, individuals with higher levels of PA $\mathrm{A}_{\text {INERTIA }}$ experienced fewer depressive symptoms. In contrast, as reported in the manuscript, individuals with higher PA $\mathrm{SD}$ reported more depressive symptoms. Also, the interaction between variability and mean level for PA predicting depressive symptoms was significant when PASD was used but was nonsignificant when PAINERTIA was used. However, despite these and other differences, the signs of most effects were in the same direction. 
Table 2

Mean Level, Autocorrelation, and Predictability Metrics of Affect Predicting Depressive Symptoms.

\begin{tabular}{|c|c|c|c|c|c|c|c|c|c|c|}
\hline & \multicolumn{10}{|c|}{ Models } \\
\hline & 1 & 2 & 3 & 4 & 5 & 6 & 7 & 8 & 9 & 10 \\
\hline $\mathrm{NA}_{\text {MEAN }}$ & $0.60 * * *$ & $0.60 * * *$ & $0.56 * * *$ & $0.58 * * *$ & $0.58 * * *$ & & & & & \\
\hline NAINERTIA & $0.06 * *$ & $0.10 * * *$ & $0.11 * * *$ & $0.11 * * *$ & $0.11 * * *$ & & & & & \\
\hline NAMEAN $*$ NAINERTIA & & $-0.19 * * *$ & $-0.19 * * *$ & $-0.21 * * *$ & $-0.10 *$ & & & & & \\
\hline NA\%DET & & & $-0.28 * * *$ & $-0.39 * * *$ & $-0.40 * * *$ & & & & & \\
\hline $\mathrm{NA}_{\mathrm{MEAN}} * \mathrm{NA} \% \mathrm{DET}$ & & & & $0.64 * * *$ & $0.61 * * *$ & & & & & \\
\hline NAINERTIA $*$ NA $\%$ DET & & & & & $0.80 * * *$ & & & & & \\
\hline 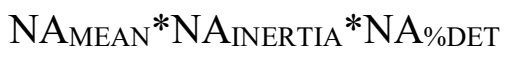 & & & & & -0.38 & & & & & \\
\hline PAMEAN & & & & & & $-0.49 * * *$ & $-0.49 * * *$ & $-0.49 * * *$ & $-0.49 * * *$ & $-0.49 * * *$ \\
\hline PAineRTIA & & & & & & $-0.09 * * *$ & $-0.08 * * *$ & $-0.09 * * *$ & $-0.09 * * *$ & $-0.09 * * *$ \\
\hline PA $_{\text {MEAN }} *$ PA INERTIA & & & & & & & 0.01 & 0.02 & 0.01 & 0.01 \\
\hline PA\%DET & & & & & & & & $-0.19 * * *$ & $-0.22 * * *$ & $-0.22 * * *$ \\
\hline $\mathrm{PA}_{\mathrm{MEAN}} * \mathrm{PA} \% \mathrm{DET}$ & & & & & & & & & $-0.24 * *$ & $-0.23 * *$ \\
\hline PAINERTIA $*$ PA\%DET & & & & & & & & & & 0.08 \\
\hline PAMEAN $*$ PAINERTIA $*$ PA $\%$ DET & & & & & & & & & & -0.34 \\
\hline Distress & 0.00 & 0.01 & -0.00 & -0.01 & -0.01 & $0.19 * * *$ & $0.19 * * *$ & $0.18 * * *$ & $0.19 * * *$ & $0.19 * * *$ \\
\hline Constant & $2.60 * * *$ & $2.60 * * *$ & $2.61 * * *$ & $2.65 * * *$ & $2.65 * * *$ & $2.35 * * *$ & $2.35 * * *$ & $2.36 * * *$ & $2.36 * * *$ & $2.36 * * *$ \\
\hline Observations & 1,297 & 1,297 & 1,297 & 1,297 & 1,297 & 1,296 & 1,296 & 1,296 & 1,296 & 1,296 \\
\hline
\end{tabular}

Note. ${ }^{*} p<.05,{ }^{*} p<.01,{ }^{* * *} p<.001$. Grey highlighting indicates findings that are different (i.e., a loss or gain of significance) from results presented in the paper using standard deviation. Underlined values represent those differences that also changed direction. 
Table 3

Mean Level, Autocorrelation, and Predictability Metrics of Affect Predicting Somatic Symptoms.

\begin{tabular}{|c|c|c|c|c|c|c|c|c|c|c|}
\hline & \multicolumn{10}{|c|}{ Models } \\
\hline & 1 & 2 & 3 & 4 & 5 & 6 & 7 & 8 & 9 & 10 \\
\hline $\mathrm{NA}_{\text {MEAN }}$ & $0.18 * * *$ & $0.18 * * *$ & $0.13 * * *$ & $0.13 * * *$ & $0.13 * * *$ & & & & & \\
\hline NAINERTIA & 0.00 & 0.01 & 0.02 & 0.02 & 0.03 & & & & & \\
\hline NAMEAN $*$ NAINERTIA & & -0.08 & -0.09 & -0.09 & -0.07 & & & & & \\
\hline NA\%DET & & & $-0.31 * * *$ & $-0.33 * * *$ & $-0.34 * * *$ & & & & & \\
\hline $\mathrm{NA}_{\text {MEAN }} * \mathrm{NA} \% \mathrm{DET}$ & & & & $0.30 *$ & $0.30 *$ & & & & & \\
\hline NAINERTIA $*$ NA\%DET & & & & & 0.02 & & & & & \\
\hline 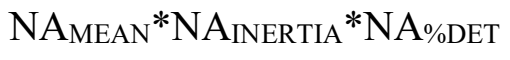 & & & & & 0.26 & & & & & \\
\hline PAMEAN & & & & & & $-0.20 * * *$ & $-0.20 * * *$ & $-0.20 * * *$ & $-0.20 * * *$ & $-0.20 * * *$ \\
\hline PA INERTIA & & & & & & 0.01 & 0.02 & 0.01 & 0.01 & 0.01 \\
\hline PAMEAN $^{*}$ PAINERTIA & & & & & & & 0.05 & 0.06 & 0.06 & 0.06 \\
\hline $\mathrm{PA} \% \mathrm{DET}$ & & & & & & & & $-0.22 * * *$ & $-0.23 * * *$ & $-0.22 * * *$ \\
\hline $\mathrm{PA}_{\text {MEAN }} * \mathrm{PA} \% \mathrm{DET}$ & & & & & & & & & -0.11 & -0.10 \\
\hline PAINERTIA $*$ PA\%DET & & & & & & & & & & 0.03 \\
\hline PAMEAN $*$ PAINERTIA $*$ PA\%DET & & & & & & & & & & -0.23 \\
\hline Distress & $0.16 * * *$ & $0.16^{* * *}$ & $0.16^{* * *}$ & $0.15 * * *$ & $0.15 * * *$ & $0.21 * * *$ & $0.21 * * *$ & $0.20 * * *$ & $0.20 * * *$ & $0.20 * * *$ \\
\hline Constant & $1.69 * * *$ & $1.69 * * *$ & $1.70 * * *$ & $1.71 * * *$ & $1.71 * * *$ & $1.63 * * *$ & $1.63 * * *$ & $1.63 * * *$ & $1.63 * * *$ & $1.63 * * *$ \\
\hline Observations & 1,286 & 1,286 & 1,286 & 1,286 & 1,286 & 1,285 & 1,285 & 1,285 & 1,285 & 1,285 \\
\hline
\end{tabular}

Note. ${ }^{*} p<.05,{ }^{*} p<.01,{ }^{* * *} p<.001$. Grey highlighting indicates findings that are different (i.e., a loss or gain of significance) from results presented in the paper using standard deviation. Underlined values represent those differences that also changed direction. 
In the PA models predicting somatic symptoms, there were a few differences when inertia as opposed to standard deviation was used as a variability metric (see highlighted coefficients in Table 3 models 6 through 10). First, PA INERTIA had no effect on somatic symptoms as compared to the effect that PASD did (see highlighted coefficients in Table 3 models 6 through 10). Second, PA\%DET did become a significant predictor of somatic symptoms (see highlighted coefficients in Table 3 models 8 through 10). Finally, the interaction term between PA $\mathrm{ANERTIA}^{*}$ PA\%DET was not a significant predictor of somatic symptoms (see Table 3 models 9 and 10) while the $\mathrm{PA}_{\mathrm{SD}} * \mathrm{PA} \% \mathrm{DET}$ was a significant predictor of somatic symptoms (as reported in the manuscript). However, despite these differences, the signs of most effects were in the same direction.

\section{Discussion}

These results suggest that emotional inertia may be different from variability given its lack of association with standard deviation or RMSSD. Indeed, Kuppens and colleagues (Kuppens et al., 2010) elaborate on the differences between emotion variability and emotional inertia. Emotional inertia is likely measuring something different from predictability as well. Instead, emotional inertia may be assessing emotional reactivity or how slowly versus quickly emotions change from one moment to the next. We also conducted the analyses with inertia replacing \%DET and found different results further bolstering the notion that inertia is also not a replacement metric for RQA predictability variables. 


\section{References}

Kuppens, P., Allen, N. B., \& Sheeber, L. B. (2010). Emotional inertia and psychological maladjustment. Psychological Science, 21, 984-991.

https://doi.org/10.1177/0956797610372634 